\title{
CANDIDATE STABILITY \\ AND VOTING CORRESPONDENCES
}

Carmelo Rodriguez-Alvarez

No 666

\section{WARWICK ECONOMIC RESEARCH PAPERS}

DEPARTMENT OF ECONOMICS

THE UNIVERSITY OF WARWICK 


\title{
Candidate Stability and Voting Correspondences ${ }^{1}$
}

\author{
Carmelo Rodríguez-Álvarez ${ }^{2}$ \\ Department of Economics \\ University of Warwick \\ CV4 7AL Coventry UK \\ C.Rodriguez-Alvarez@warwick.ac.uk
}

This version: January 2003

\footnotetext{
${ }^{1}$ This paper is a revised version of the second chapter of my Ph.D. Dissertation. I am indebted to my supervisor Salvador Barberà for his advice, encouragement and patience. I also thank Dolors Berga, Carmen Beviá, Walter Bossert, Jernej Čopič, Bhaskar Dutta, Matt Jackson, Jordi Massó, Diego Moreno, David Pérez-Castrillo and Yves Sprumont for their helpful comments and suggestions. Financial support through Research Grant 1998 FI 00022 from Comissionat per Universitats i Recerca, Generalitat de Catalunya and Research Project PB98-870 from Ministerio de Ciencia y Tecnología is gratefully acknowledged. This work was completed at the University of Warwick, I would like to thank the hospitality of the Department of Economics and the financial support from Fundación Barrié de la Maza. Of course, I am solely responsible for all the remaining errors.

${ }^{2}$ Phone: $+(44)(0) 2476523479$. Fax:+(44)(0) 24765 23032. URL: http://www.warwick.ac.uk/ ẽcrcal.html.
} 


\begin{abstract}
For each set of candidates at stake and each profile of voters' preferences over running candidates a voting correspondence selects a set of candidates. Following Dutta, Jackson and Le Breton (Econometrica, 2001), a voting correspondence is candidate stable if a candidate never benefits from withdrawing unilaterally her candidacy. If candidates cannot vote and compare sets of candidates according to their expected utility conditional on some prior probability assessment only dictatorial voting correspondences are candidate stable and unanimous. If the assessments are restricted to be uniform, rules that select the set of best candidates of two fixed voters are also allowed. We also analyze other domains of preferences fitting extreme attitudes towards risk in which positive results are obtained.
\end{abstract}

JEL Classification Number: D71.

Keywords: Voting Correspondence, Candidate Stability, Preferences over Sets. 


\section{Introduction}

The decision of the candidates to enter the fray is of capital importance for the result of any public choice problem. In fact, there are circumstances in which candidates can influence the result of the social choice by leaving the fray, even when they have no chance of winning. For instance, we can think of a presidential election in which there are three candidates, one of them conservative, with the support of $40 \%$ of the voters, and two of them leftist, with the support of $35 \%$, and $25 \%$, respectively. If the winner is decided by plurality and voters cast their ballot according to their true preferences, the conservative candidate wins, but if instead one of the leftist candidates quits, the other leftist candidate is chosen. If the leftist candidates prefer a leftist president to a conservative one, they will have incentives to exit the poll and transfer their votes to the other leftist candidate.

An election can be modeled as two-stage process. In a first stage, a set of candidates decide whether they run the election or they leave the ballot. Later on, voters decide the social outcome using a voting rule that takes into account their preferences over the running candidates. The Social Choice literature has devoted a considerable amount of interest to analyze the incentives of the voters to misrepresent their preferences. However, it has been usually assumed that the set of candidates (or possible alternatives) is exogenous and independent of any individual decision. In this paper, we analyze the incentives of the candidates in the first stage of the election. We investigate the features of voting rules that make the candidates not to prefer to exit the election unilaterally rather than to run the fray. In other terms, under such voting rules staying as a candidate when the rest of the candidates stay is always a Nash Equilibrium strategy for all the candidates. This condition is called "candidate stability". Only if candidate stability holds we can consider the set of feasible candidates as exogenous.

The analysis of candidates' strategic concerns is a relatively new topic in the Social Choice literature. Osborne and Slivinski [17] and Besley and Coate [5] were among the first who studied the problem of strategic candidacy in representative democracy models. They focused on the number of candidates and the pattern of entry of candidates in elections ruled by plurality. We instead follow the normative approach proposed by Dutta, Jackson and Le Breton $[10],{ }^{1}$ who 
introduced candidate stability condition in single-valued elections. Their main result is that any single-valued unanimous and candidate stable voting procedure must be dictatorial if candidates are not allowed to vote. In the case of voting candidates, they also show that an impossibility result holds for a large family of voting rules.

In the present work, we generalize DJL's analysis to multivalued environments. Therefore, we model elections as voting correspondences. For each set of candidates at stake and each preference profile over candidates in the ballot of voters, a voting correspondence chooses a set of candidates. We can interpret a voting correspondence as a first screening device that narrows the social agenda to a smaller set of candidates. Candidates do know this first selection but they are not aware of the final resolution of the social choice. Therefore, candidates consider the selected set as the result of their stay-quit decision.

Another possible interpretation of multivalued social choice fits scenarios in which voters play a voting mechanism that admits multiple equilibria at some profile of voters' preferences. We can think of situations in which the candidates know the set of equilibria of the voting mechanism, but they do not have enough information to deduce which are the strategies actually played by the voters and the equilibrium that eventually arises. In these cases, the candidates cannot apply any backward induction argument to focus on a unique equilibrium. Nevertheless, they may consider the whole set of possible equilibria as the result of the social choice.

When the result of the social choice is multivalued, the study of the candidates' strategic concerns becomes problematic. Individuals are naturally endowed with preferences over candidates, but such preferences do not contain enough information to compare sets of candidates. Thus, the candidates must be endowed with preferences over sets of alternatives. These preferences are going to be naturally restricted. They must be consistent not only with the preferences over single candidates, but they should also depend on the interpretation attached to the notion of sets; that is, on how the candidates think that the ties are going to be resolved. In this work we propose different restricted domains of preferences over sets that fit different interpretations of the resolution of the social choice. For instance, we can suppose that candidates compare sets according to their expected utility. Alternatively, they could rely on some criterion consistent

\footnotetext{
${ }^{1}$ Henceforth DJL.
} 
with extreme attitudes towards risk, and their preferences may be consistent with leximin, maximin or maximax criteria. ${ }^{2}$ Indeed, the implications of candidate stability will depend crucially on the choice of the specific domain of preferences over sets.

We prove that if candidates are not allowed to vote and they compare sets according to their expected utility conditional on some prior probability assessment, only dictatorial rules are candidate stable and unanimous. If the prior assessments are restricted to be uniform, also rules that select the best candidates for two fixed agents satisfy both conditions. We obtain less pessimistic results if candidates' preferences over sets are consistent with extreme attitudes towards risk.

Also related works are Ehlers and Weymark [11] and Eraslan and McLennan [12]. These two papers and ours are independent but complementary contributions extending the DJL's model to set valued elections. Both Ehlers et al. [11] and Eraslan et al. [12] propose the same condition of candidate stability, but they do not model explicitly the role of preferences of candidates over sets of candidates. Hence, they restrict attention to only one of the possible scenarios considered here. In Ehlers et al. [11] an alternative direct proof of the main theorem of DJL is provided. They also show that the arguments in their proof can be applied to multivalued environments and they obtain that only dictatorial rules are candidate stable and unanimous. In Eraslan et al. [12] DJL's framework is also extended to allow the voters to report indifferences. They prove that only serial dictatorships satisfy candidate stability and unanimity.

A companion paper, Rodríguez-Álvarez [20], proposes a probabilistic framework for the analysis of candidates' strategic concerns. In this paper, elections are modeled as probabilistic voting procedures, which are rules that for each agenda and each profile of preferences select a lottery on the set of candidates. In the probabilistic framework, the implications of candidate stability depend crucially on the size of the set of candidates. Not surprisingly, random combinations of dictatorial rules are always unanimous and candidate stable. Nevertheless, many other possibil-

\footnotetext{
${ }^{2}$ These domains of preferences over sets have been applied to the study of strategy-proofness of social choice correspondences in Pattanaik [18] and [19], Gärdenfors [13], Barberà, Dutta and Sen [3], and Campbell and Kelly [6] among others. We refer the reader to Barberà , Bossert and Pattanaik [2] for a recent survey on ranking sets of objects.
} 
ities can be devised. For instance, when there are only three candidates a rule assigning equal probability to the set of efficient candidates at stake is unanimous and candidate stable.

In a different vein, we can mention the work of Sönmez [23] who studies the incentives of the agents to affect the social agenda in a particular multivalued economic environment: centralized two sided matching markets. Specifically, he focuses on the incentives of a hospital to destroy positions available to medical interns and he shows that the solution used to match students and hospitals in the US is manipulable in this way. The main difference with our analysis relies on his interpretation of multiple outcomes. A hospital may be interested in hiring more than one medical intern. So, the students matched to each hospital are compatible and there is no need to solve possible ties. Hence, hospitals' preferences over sets of interns do not fit the domains of preferences over sets that we analyze here.

Finally, we refer to an interesting paper by Berga, Bergantiños, Massó and Neme [4], who also analyze the incentives of the participants in a multivalued election to withdraw. They study the problem of a society choosing a subset of new members from a finite set of candidates. They consider explicitly the possibility that the founding members of the society may want to leave it if they do not like the resulting new society. It is shown that only unanimity rule is nonmanipulable and stable according to their definition. In contrast to candidate stability, their stability condition is not strategic. In their framework, when considering exiting, each agent does not take into account the effect of her withdrawal on the social outcome. Instead, she may consider withdrawing because she may prefer to stay alone rather than to belong to the resulting new society.

The remainder of the paper is structured as follows. We devote Section 2 to outline the setting and provide definitions and notation, and Section 3 to develop the implications of candidate stability when candidates are expected utility maximizers and are not allowed to vote. In Section 4 we refer to the possibilities that arise when the candidates are reluctant to express strict preference among sets, or their preferences over sets are consistent with extreme attitudes towards risk. Finally, in Section 5 we discuss the possible overlap between candidates and voters' sets. In Appendix 1 we include the proofs of the theorems. In Appendix 2 we prove some intermediate results. 


\title{
2 Definitions
}

\subsection{Candidates, Voters and Preferences}

\author{
Voters and Candidates
}

Let $\mathcal{N}$ be a society formed by a finite set of voters $\mathcal{V}$, and a finite set of candidates $\mathcal{C}$, $\mathcal{N}=\mathcal{C} \cup \mathcal{V}$. We consider the case in which $\# \mathcal{C} \geq 3$ since the strategic concerns with $\# \mathcal{C} \leq 2$ are trivial. ${ }^{3}$ We focus on the case in which there is no overlap between the sets of voters and candidates, $\mathcal{C} \cap \mathcal{V}=\{\varnothing\}$. It could be an approximation to the situation in which the set of candidates is relatively small with respect to the set of voters, and hence, their capability to influence the social choice is negligible. Moreover, in this scenario, we can isolate the incentives of the candidates to participate in an election, regardless of their concerns as voters. ${ }^{4}$

\section{Preferences over Candidates}

Let $\mathcal{P}$ be the set of all linear orders on $\mathcal{C} \cup\{\varnothing\}$, where the empty set refers to the situation in which no candidate is elected. Each individual $i \in \mathcal{N}$ is endowed with a linear order $P_{i} \in \mathcal{P} .^{5}$ Let $\mathcal{P}^{i} \subset \mathcal{P}$ be the set of preferences over candidates admissible for individual $i$. We assume that for all $i$, any candidate is preferred to the empty set. Preferences of voters over candidates are not restricted in any other way. On the other hand, each candidate considers herself as the best alternative. That is, $a P_{a} b$ for all $a, b \in \mathcal{C}, P_{a} \in \mathcal{P}^{a}$. We denote by $P \in \mathcal{P}^{\mathcal{V}}$ a voters' preference profile.

Let $2^{\mathcal{C}} \backslash\{\varnothing\}$ be the set of all non-empty finite subsets of $\mathcal{C}$. We refer to an arbitrary element of $2^{\mathcal{C}} \backslash\{\varnothing\}$ as an agenda. For each $A \in 2^{\mathcal{C}} \backslash\{\varnothing\},\left.P\right|_{A}$ denotes the restriction of $P$ to the set $A$. By top $\left(A, P_{i}\right)$ and bottom $\left(A, P_{i}\right)$ we mean, respectively, the best and the worst element on $A$ according to the order $P_{i} \in \mathcal{P}^{i}$.

\footnotetext{
${ }^{3} \# A$ refers to the cardinality of the set $A$.

${ }^{4}$ When candidates can be voters, the analysis of stability becomes more complicated because candidates' preferences are assumed to favor their own election. Then, stronger conditions are required and their interpretation is not clear in terms of candidates' incentives. This interesting case is left to Section 5 .

${ }^{5} \mathrm{~A}$ linear order is a complete, antisymmetric and transitive binary relation. For each $P_{i} \in \mathcal{P}$, the associated weak preference relation is denoted by $R_{i}$.
} 
Abusing notation, for any set $I \subseteq \mathcal{V}, \mathcal{P}^{I}$ is defined as the set of admissible preferences profiles for $I$. Then, for any $P \in \mathcal{P}^{\mathcal{V}}, P_{I} \in \mathcal{P}^{I}$ refers to the restriction of the profile $P \in \mathcal{P}^{\mathcal{V}}$ to the members of $I$.

\section{Preferences over Sets of Candidates}

As we have already noted, we are interested in the incentives of candidates to withdraw from multivalued elections. Then, in order to compare social outcomes, candidates must be endowed with preferences over sets of candidates.

Let $\mathcal{D}$ be the set of all complete and reflexive binary relations on $2^{\mathcal{C}} \backslash\{\varnothing\}$. We denote by $\succsim \in \mathcal{D}$ an arbitrary preference relation over sets of candidates. Although we assume strict preferences over alternatives, we do not rule out the possibility of indifference among sets. Hence for each $\succsim \in \mathcal{D}, \succ$ refers to the strict component of $\succsim$, while $\sim$ refers to the indifference term that is immediately defined from $\succ$ in the standard manner.

The candidates' preferences over sets are naturally restricted. They must be consistent with the restriction on their preferences over single candidates and our interpretation of sets of candidates. According to the different interpretations in the resolution of the social choice we present several environments which define different domains of admissible preferences over sets. We will defer the presentation of the different environments and domains until later sections. At this point, we introduce some notation.

For any $a \in \mathcal{C}$, the domain $\mathcal{D}_{a}^{\mathcal{E}} \subset \mathcal{D}$ denotes the set of admissible preferences over sets of candidates for candidate $a$ in the environment $\mathcal{E}$.

Equivalently, we can define $\mathcal{D}_{a}^{\mathcal{E}}$ via the admissible preferences over single candidates. Given the environment $\mathcal{E}$, for any $P_{a} \in \mathcal{P}^{a}$, let $\succsim^{\mathcal{E}}\left(P_{a}\right) \subset \mathcal{D}$ be the set of admissible preferences over sets given $P_{a}$ in the environment $\mathcal{E}$. Then $\mathcal{D}_{a}^{\mathcal{E}}$ could be defined in the following way:

$$
\mathcal{D}_{a}^{\mathcal{E}}=\left\{\succsim \in \mathcal{D} \text { s.t. there is } P_{a} \in \mathcal{P}^{a} \text { with } \succsim \in \succsim^{\mathcal{E}}\left(P_{a}\right)\right\} \text {. }
$$




\subsection{Voting Correspondences}

Definition 1. A voting correspondence $v$ is a mapping $v: 2^{\mathcal{C}} \backslash\{\varnothing\} \times \mathcal{P}^{\mathcal{V}} \rightarrow 2^{\mathcal{C}} \backslash\{\varnothing\}$ such that for all $A \in 2^{\mathcal{C}} \backslash\{\varnothing\}$ and for all $P \in \mathcal{P}^{\mathcal{V}}$ :

i) $v(A, P) \subseteq A$

ii) $v(A, P)=v\left(A, P^{\prime}\right)$ for all $P^{\prime} \in \mathcal{P}^{\mathcal{V}}$ such that, $\left.P\right|_{A}=\left.P^{\prime}\right|_{A}$.

Item $i$ ) refers to the fact that candidates cannot be selected if they are not at stake.

In item $i i)$ we assume Arrow's Independence of Irrelevant Alternatives; that is, only the preferences of voters over the candidates at stake matter.

Notice that a voting correspondence is a collection of social choice correspondences, one for each configuration of the agenda. A voting correspondence is more general than a voting procedure as defined in DJL, since a voting correspondence allows for multivalued choices.

\subsection{Candidate Stability}

In this paper we investigate the possibility of constructing voting correspondences for which the initial agenda $\mathcal{C}$ can be considered exogenous and independent of the preferences of the voters. An agenda can be considered exogenous if the running candidates cannot improve by leaving the electoral fray and the candidates who are not at stake have no incentives to enter the election. Although it seems immediate to introduce a stability condition for candidates who are in the ballot, it is not clear which would be a correct statement of stability regarding the incentives of the non-running candidates. That is the reason why we focus on the stability of the initial agenda in which no additional candidate can enter in the fray. However, we will see that the stability condition over the full agenda, candidate stability, is rather powerful and allows us to state clear results.

We say a voting correspondence $v$ is candidate stable if and only if for all $a \in \mathcal{C}$ and for all $P \in \mathcal{P}^{\mathcal{V}}, a$ does not prefer $v(\mathcal{C} \backslash\{a\}, P)$ to $v(\mathcal{C}, P)$. Equivalently, a voting correspondence is candidate stable if and only if the entry of all candidates always constitutes a Nash equilibrium strategy for all candidates. 
Candidate stability is not a condition on the entry-exit game form, but on the family of games which are generated from the entry-exit game form once the preferences of agents over social choice outcomes are specified. Since the result of the entry-exit decision by the candidates is not necessarily a singleton, these preferences cannot be trivially derived from candidates' preferences over candidates. Hence, candidates have to be endowed with some criterion in order to extend their preferences from alternatives to sets of alternatives. In order for the Nash equilibrium requirements not to be trivially fulfilled, we have assumed that preferences over sets of candidates are complete although not necessarily transitive. ${ }^{6}$

The choice of the domain restriction on candidates' preferences over sets will become crucial for our analysis since the different domains lead to different implications of candidate stability. We now include a generic definition of candidate stability in an arbitrary environment $\mathcal{E}$.

Definition 2. A voting correspondence $v$ is candidate stable in the environment $\mathcal{E}$ (or $\mathcal{E}$ candidate stable) if and only if for all $a \in \mathcal{C}$, for all $P \in \mathcal{P}^{\mathcal{V}}$ and for all $\succsim_{a} \in \mathcal{D}_{a}^{\mathcal{E}}, v(\mathcal{C}, P) \succsim_{a}$ $v(\mathcal{C} \backslash\{a\}, P)$.

We can provide different definitions of stability while maintaining the same spirit of candidate stability. For instance, we could investigate rules for which any possible agenda were stable. That is, candidates should never have incentives to leave the fray independently of the configuration of the ballot. With this notion of stability at hand, we would introduce the logic of candidate stability at any possible agenda $A \in 2^{\mathcal{C}} \backslash\{\varnothing\}$. Evidently, such condition would be a stronger one than candidate stability, but we would obtain the same characterization results (up to minimal modifications in the statement). Although this new stability condition may facilitate the proofs and the presentation of the results, we follow DJL formulation of the stability condition, which allows us to state stronger results.

\subsection{Unanimity}

In this work we abstract from the mechanism used in the voting stage. We only assume unanimity, which is uncontroversial. When all voters agree on who is the best candidate, this is

\footnotetext{
${ }^{6}$ This is the case if candidates are reluctant to express strict preferences among sets. This possibility is analyzed in Section 4.
} 
elected.

Definition 3. The voting correspondence $v$ satisfies unanimity if for any $B \in\left\{\mathcal{C},\left\{\mathcal{C} \backslash\{a\}_{a \in \mathcal{C}}\right\}\right\}$, $b \in B$, and all $P \in \mathcal{P}^{\mathcal{V}}, b=\operatorname{top}\left(B, P_{i}\right)$ for all $i \in \mathcal{V}$ implies $v(B, P)=b$.

We employ a weak version of unanimity since candidate stabilty only applies to agendas in which no more than a single candidate withdraws.

\section{Candidate Stability and Conditional Expected Utility Maxi- mizers}

We begin the formal analysis by introducing two domains of preferences over sets that match scenarios in which the candidates are expected utility maximizers. These domains of preferences were proposed in Barberà et al. [3] for the study of strategy-proof social choice correspondences. In these environments, candidates are assumed to interpret the possible social choice outcomes as the basis of lotteries and to have von Neumann-Morgenstern preferences over lotteries. If a set is chosen, candidates attribute some probabilities of choice to the elements of this. The proposed scenarios differ in the description of the way individuals will assess the probabilities associated to sets. In the first one, Conditional Expected Utility Consistent Preferences(or simply, BDS1), each candidate is assumed to subjectively assess one probability distribution over $\mathcal{C}$ and to evaluate each subset according to its conditional expected utility. In the second scenario, Conditional Expected Utility Consistent with Even Chance Probabilities Preferences(BDS2), the initial distribution of probability is restricted to be uniform, all candidates associate each set with an even chance lottery over all its components. We now state this formally.

A utility function is a mapping $u: \mathcal{C} \longrightarrow \mathbb{R}$. We say that $u$ fits preference ordering $P \in \mathcal{P}$ or represents $P$ if for any $a, b \in \mathcal{C}, a P b$ if and only if $u(a)>u(b)$. An individual probability assessment $\lambda_{i}$ is a function $\lambda_{i}: \mathcal{C} \longrightarrow(0,1)$, such that $\sum_{a \in \mathcal{C}} \lambda_{i}(a)=1$. We rule out degenerate lotteries as probability assessments since those are not consistent with our interpretation of sets of candidates as the possible results of the social choice process.

Definition 4 (Conditional Expected Utility Consistent; BDS1). The ordering $\succsim \in \mathcal{D}$ 
is a Conditional Expected Utility Consistent ordering admissible for candidate $a \in \mathcal{C}$, we write $\succsim \in \mathcal{D}_{a}^{1}$, if and only if there is $P_{a} \in \mathcal{P}^{a}$, utility function $u_{a}$ representing $P_{a}$ along with a prior assessment $\lambda_{a}$ such that for all $X, Y \in 2^{\mathcal{C}} \backslash\{\varnothing\}$ :

$$
X \succsim Y \Leftrightarrow \sum_{x \in X} \frac{\lambda_{a}(x) u_{a}(x)}{\left(\sum_{x^{\prime} \in X} \lambda_{a}\left(x^{\prime}\right)\right)} \geq \sum_{y \in Y} \frac{\lambda_{a}(y) u_{a}(y)}{\left(\sum_{y^{\prime} \in Y} \lambda_{a}\left(y^{\prime}\right)\right)} .
$$

\section{Definition 5 (Conditional Expected Utility Consistent with Equal Probabilities;}

BDS2). The ordering $\succsim \in \mathcal{D}$ is a Conditional Expected Utility Consistent with Equal Probabilities ordering admissible for candidate $a \in \mathcal{C}, \succsim \in \mathcal{D}_{a}^{2}$, if and only if there is $P_{a} \in \mathcal{P}^{a}$ and a utility function $u_{a}$ fitting $P_{a}$ such that for all $X, Y \in 2^{\mathcal{C}} \backslash\{\varnothing\}$ :

$$
X \succsim Y \Leftrightarrow \frac{1}{\# X} \sum_{x \in X} u_{a}(x) \geq \frac{1}{\# Y} \sum_{y \in Y} u_{a}(y) .
$$

Remark 1. BDS2 preferences are a special case of BDS1 preferences, where prior assessments are restricted to be uniform. Then, $\mathcal{D}_{a}^{2} \subset \mathcal{D}_{a}^{1}$.

Before proceeding to state the restrictions that candidate stability implies in both environments, we begin with a lemma on the existence of various admissible preference orderings in $\mathcal{D}_{a}^{1}$ and $\mathcal{D}_{a}^{2}$. Its proof appears in Appendix 2.

Lemma 1. Let $a \in \mathcal{C}$ and $X, Y \in 2^{\mathcal{C}} \backslash\{\varnothing\}$ :

i) If $a \notin(X \cup Y)$ then there exists $\succsim \in \mathcal{D}_{a}^{2}$ such that $X \succ Y$.

ii) If $X, Y$ are such that $X=(Y \cup\{a\})$ and $a \notin Y$, then for all $\succsim \in \mathcal{D}_{a}^{1} X \succ Y$.

iii) If $a \in Y, Y \neq(X \cup\{a\})$ and $\# Y \neq 1$, then there is $a \succsim \in \mathcal{D}_{a}^{1}$ such that $X \succ Y$.

iv) If there is $b \in Y$ such that $(X \backslash\{a\})=(Y \backslash\{b\})$, then for all $\succsim \in \mathcal{D}_{a}^{2} X \succ Y$.

v) If $a \in Y, \# Y \neq 1$ and for all $b \in(X \cup\{\varnothing\}),(X \backslash\{b\}) \neq(Y \backslash\{a\})$, then there exists $\succsim \in \mathcal{D}_{a}^{2}$ such that $X \succ_{a}^{2} Y$.

Lemma 1 presents the main features of the admissible preferences over sets in both BDS domains. Items i), iii) and v) imply that the preferences of the candidates over a significant 
amount of sets are not restricted. Moreover, item iv) says that the BDS2 criterion generates responsive preferences: for any two sets that differ in only one candidate, the set containing the most desirable candidate is always preferred.

Now, we can state the implications of candidate stability in both BDS environments. In single-valued elections, as a result of the self-preference of the candidates, DJL have proved that the incentives to withdraw only affect the non-elected candidates. In multivalued voting processes this fact no longer holds, as we see in the following lemmata.

Lemma 2. A voting correspondence $v$ is BDS1 candidate stable if and only if for all $a \in \mathcal{C}$ and all $P \in \mathcal{P}^{\mathcal{V}}$ either $v(\mathcal{C}, P)=\{a\}$ or:

i) if $a \in v(\mathcal{C}, P)$ and $v(\mathcal{C}, P) \neq\{a\}$, then $v(\mathcal{C}, P)=v(\mathcal{C} \backslash\{a\}, P) \cup\{a\}$;

ii) if $a \notin v(\mathcal{C}, P)$, then $v(\mathcal{C}, P)=v(\mathcal{C} \backslash\{a\}, P)$.

Proof. We start by proving sufficiency. Consider an arbitrary candidate $a \in \mathcal{C}$ and an arbitrary profile of voters preferences $P \in \mathcal{P}^{\mathcal{V}}$. Assume first that $v(\mathcal{C}, P)=\{a\}$. By the self preference of the candidates, for all $\succsim \in \mathcal{D}_{a}^{1}, v(\mathcal{C}, P) \succsim v(\mathcal{C} \backslash\{a\}, P)$, and the candidate $a$ has no incentive to leave the fray. Suppose now that $a \in v(\mathcal{C}, P), v(\mathcal{C}, P) \neq\{a\}$ and $(v(\mathcal{C}, P)=v(\mathcal{C} \backslash\{a\}, P) \cup\{a\})$, by item ii) of Lemma 1 , we know that for all $\succsim \in \mathcal{D}_{a}^{1}, v(\mathcal{C}, P) \succ v(\mathcal{C} \backslash\{a\}, P)$. Finally, if $a \notin v(\mathcal{C}, P)$ and $v(\mathcal{C}, P)=v(\mathcal{C} \backslash\{a\}, P)$, then the candidate $a$ 's withdrawal does not affect the social choice so she is not better off after leaving the fray. This case exhausts the possibilities, and as the choice of $P$ was arbitrary, sufficiency holds.

Now we check necessity. First, let us prove i). Assume to the contrary that $v$ is BDS1 candidate stable but there are $a \in \mathcal{C}$, and $P \in \mathcal{P}^{\mathcal{V}}$ such that $a \in v(\mathcal{C}, P), v(\mathcal{C}, P) \neq\{a\}$ and $v(\mathcal{C}, P) \neq(v(\mathcal{C} \backslash\{a\}, P) \cup\{a\})$. By item iii) of Lemma 1 , we know there is $\succsim \in \mathcal{D}_{a}^{1}$ such that $v(\mathcal{C} \backslash\{a\}, P) \succ v(\mathcal{C}, P)$, which contradicts BDS1 candidate stability. In order to check the necessity of ii), assume again that $v$ is BDS1 candidate stable but there are $a \in \mathcal{C}, P \in \mathcal{P}^{\mathcal{V}}$ such that $a \notin v(\mathcal{C}, P)$, but $v(\mathcal{C}, P) \neq v(\mathcal{C} \backslash\{a\}, P)$. Notice that, $a \notin(v(\mathcal{C}, P) \cup v(\mathcal{C} \backslash\{a\}, P))$, hence by item i) of Lemma 1 and Remark 1 , there is $\succsim \in \mathcal{D}_{a}^{1}$ with $v(\mathcal{C} \backslash\{a\}, P) \succ v(\mathcal{C}, P)$, again contradicting BDS1 candidate stability. 
Lemma 2 shows that in the domain of BDS1 preferences, if an elected candidate quits the ballot then the social choice after her withdrawal must be the election of the remaining previously elected candidates. Moreover, the withdrawal of a non-elected candidates must have no effect on the social choice. Lemma 2 provides an expected utility rationale for the strong candidate stability condition proposed in Ehlers and Weymark [11] and Eraslan and McLennan [12]. Only when the candidates cannot vote and compare sets according to Conditional Expected Utility Consistent preferences, the strong candidate stability condition introduced in these works is equivalent to a candidate stability condition derived from candidates preferences over the possible social choice outcomes.

Lemma 3. A voting correspondence $v$ is BDS2 candidate stable if and only if for all $a \in \mathcal{C}$ and all $P \in \mathcal{P}^{\mathcal{V}}$ either $v(\mathcal{C}, P)=\{a\}$ or:

i) if $a \in v(\mathcal{C}, P)$ and $v(\mathcal{C}, P) \neq\{a\}$, then either:

a) $v(\mathcal{C}, P)=v(\mathcal{C} \backslash\{a\}, P) \cup\{a\}$ or

b) there is $b \in \mathcal{C} \backslash\{a\}$ such that $v(\mathcal{C} \backslash\{a\}, P)=(v(\mathcal{C}, P) \backslash\{a\}) \cup\{b\}$;

ii) if $a \notin v(\mathcal{C}, P)$, then $v(\mathcal{C}, P)=v(\mathcal{C} \backslash\{a\}, P)$.

Proof. In order to prove sufficiency, we only have to check item $i, b)$ since, as for all $a \in \mathcal{C}$ $\mathcal{D}_{a}^{2} \subset \mathcal{D}_{a}^{1}$, the arguments in Lemma 2 apply directly to the remaining cases. Hence, we consider $P \in \mathcal{P}^{\mathcal{V}}$ and $a \in v(\mathcal{C}, P)$ such that there is another candidate $b \in \mathcal{C}$ satisfying $v(\mathcal{C} \backslash\{a\}, P)=$ $\left(v(\mathcal{C}, P) \backslash\{a\} \cup\{b\}\right.$. By the responsiveness of the preferences in $\mathcal{D}_{a}^{2}$ (item iv) of Lemma 1), we have that for all $\succsim \in \mathcal{D}_{a}^{2}, v(\mathcal{C}, P) \succ v(\mathcal{C} \backslash\{a\}, P)$. So, BDS2 candidate stability holds.

Now, we prove the necessity. Assume to the contrary that $v$ is BDS2 candidate stable but it does not satisfy item i). Hence, there are $a \in v(\mathcal{C}, P)$ and $P \in \mathcal{P}^{\mathcal{V}}$ such that there is no candidate $b \in(v(\mathcal{C} \backslash\{a\}, P) \cup\{\varnothing\})$, such that $v(\mathcal{C} \backslash\{a\}, P)=(v(\mathcal{C}, P) \backslash\{a\}) \cup\{b\}$. Applying item v) of Lemma 1 , we know there is $\succsim \in \mathcal{D}_{a}^{2}$ such that $v(\mathcal{C} \backslash\{a\}, P) \succ v(\mathcal{C}, P)$, which contradicts BDS2 candidate stability. Finally, the arguments in the proof of necessity of Lemma 2 can also be applied in the BDS2 environment in order to show the necessity of item ii). 
Even though BDS2 restricts considerably the preferences of the candidates, BDS2 candidate stability is not much weaker than BDS1 candidate stability. Basically, the only new possibility that arises with respect to the BDS1 environment is that an elected candidate does not have incentives to leave the fray if her withdrawal results in the selection of another candidate.

In the following Theorem 1 and Theorem 2, we show that candidate stability and unanimity introduce rather compelling restrictions in both BDS scenarios. Almost any unanimous voting correspondence is subject to the incentives of the candidates to leave the ballot in environments in which sets of candidates are compared according to the postulates of the Expected Utility Theory. Before we state the characterization results, we need to introduce some notation.

A voting correspondence $v$ is dictatorial if there exists a voter $i \in \mathcal{V}$ such that $v(A, P)=$ top $\left(A, P_{i}\right)$ for all $P \in \mathcal{P}^{\mathcal{V}}$ and all agendas $A \in\left\{\mathcal{C},\{\mathcal{C} \backslash\{a\}\}_{a \in \mathcal{C}}\right\}$. Analogously, a voting correspondence $v$ is bidictatorial if there are two voters $i, j \in \mathcal{V}$ such that $v(A, P)=\operatorname{top}\left(A, P_{i}\right) \cup \operatorname{top}\left(A, P_{j}\right)$ for all $P \in \mathcal{P}^{\mathcal{V}}$ and all agendas $A \in\left\{\mathcal{C},\{\mathcal{C} \backslash\{a\}\}_{a \in \mathcal{C}}\right\} .{ }^{7}$ Finally, for any $I \subseteq \mathcal{V}, P \in \mathcal{P}^{\mathcal{V}}$ and $A \in 2^{\mathcal{C}} \backslash\{\varnothing\}$, we define the Pareto Correspondence associated to voters $I$ and agenda $A$ as Pareto $\left(A, P_{I}\right)=\left\{a \in A\right.$, s.t. for no $b \in A, b P_{i} a$ for all $\left.i \in I\right\}$. We say that a candidate $a$ is efficient with respect to agenda $A$ and preference profile $P$ if $a \in \operatorname{Pareto}(A, P)$. Conversely, a candidate $b$ is Pareto dominated with respect to agenda $A$ and profile $P$ if $b \in A$ but $b \notin \operatorname{Pareto}(A, P)$.

Theorem 1. A voting correspondence $v$ is unanimous and BDS1 candidate stable if and only if it is dictatorial.

Theorem 1 is equivalent to the main theorem in Eraslan et al. [12] when candidates cannot vote and voters are not allowed to express weak preferences. Our Theorem 1 also tells us that the result in DJL's Theorem 1 also applies to multivalued environments if the candidates preferences over sets of candidates are not severely restricted.

\footnotetext{
${ }^{7}$ This restricted definition of dictatorial rules is due to the fact that we only care about the stability of the whole set of candidates.
} 
Theorem 2. Let $v$ be a unanimous voting correspondence.

i) Assume that $\# \mathcal{C} \geq 4$; then $v$ is candidate stable according to BDS2 if and only if it is dictatorial or bidictatorial.

ii) Assume that $\# \mathcal{C}=3 ; v$ is candidate stable according to BDS2 if and only if there is a set of voters $S \subseteq \mathcal{V}$, such that for all $A \in\left\{\mathcal{C},\{\mathcal{C} \backslash\{a\}\}_{a \in \mathcal{C}}\right\}$ and for all $P \in \mathcal{P}^{\mathcal{V}}$ :

a) $v(A, P) \subseteq \operatorname{Pareto}\left(A, P_{S}\right)$ and,

b) for any $a, b \in \mathcal{C}, v(A, P) \neq\{a\}$ if there is $i \in S$ such that $b P_{i} a$.

In the light of Lemma 1 and Lemma 3, the results of Theorem 2 are not surprising. In general, only rules that choose the best candidates of two arbitrarily fixed voters are allowed apart from dictatorial ones. However, when the initial set of candidates is fairly small another possibilities arise. In this case the self preference of the candidates and the responsiveness of the preferences reduce considerably the set of admissible preferences and candidates' incentives to withdraw.

The proofs of Theorem 1 and Theorem 2 are in Appendix 1. The proofs rely on the arguments of a more general result, Theorem 4, that appears in Section 5. Theorem 4 allows for an overlap between the candidates' and voters' sets at the cost of employing stability notions that are not directly derived from the candidates' incentives to withdraw the election. We provide now a sketch of the proofs. We analyze first the election when voters preferences are restricted to prefer three arbitrary candidates to the rest and voters also agree in their preferences over the remaining candidates. In this restricted environment, a candidate stable and unanimous voting correspondence can be rationalizable by an auxiliary group decision rule. In both conditional expected utility scenarios, candidate stability and unanimity imply that the auxiliary group decision rule always generates quasitransitive orderings on $\mathcal{C},{ }^{8}$ and satisfies Unrestricted Domain, Pareto Efficiency and Arrow's Independence of Irrelevant Alternatives. Hence, we can apply a version of Arrow's Theorem stated by Mas-Colell and Sonnenschein [14] to obtain that there is a group of voters holding veto power over $v$. Then, the joint implications of unanimity and

\footnotetext{
${ }^{8} \mathrm{~A}$ binary relation is quasitransitive if its strict component is transitive.
} 
candidate stability allow us to extended to arbitrary profiles of preferences. Moreover, in the BDS1 scenario, it is immediate to see that only one voter may hold veto power and then the dictatorship result is obtained. ${ }^{9}$

In the BDS2 environment, we need at least four candidates to get the bidictatorship result. When, there are only three candidates, many rules satisfy the requirements of unanimity and BDS2 candidate stability. We can mention the correspondences choosing the top candidates of an arbitrarily large fixed group of voters, or the Pareto correspondence. Nevertheless, many other rules fulfill the conditions of Theorem 2.

Example 1. Let $\mathcal{V}=\{1, \ldots, \# \mathcal{V}\}$, and $\# \mathcal{C}=3$. For any $P \in \mathcal{P}^{\mathcal{V}}$ and $A \in 2^{\mathcal{C}} \backslash\{\varnothing\}$ define by $k^{*}(A, P)=\min \left\{i \in \mathcal{V}\right.$, s.t. $\left.\operatorname{top}\left(A, P_{i}\right) \neq \operatorname{top}\left(A, P_{1}\right)\right\}$. Construct the voting correspondence $\tilde{v}$ in the following way, for all $A \in 2^{\mathcal{C}} \backslash\{\varnothing\}$, and for all $P \in \mathcal{P}^{\mathcal{V}}, \tilde{v}(A, P)=\operatorname{top}\left(A, P_{1}\right) \cup$ top $\left(A, P_{k^{*}(A, P)}\right)$. Notice that $\tilde{v}$ satisfies $\left.\left.\boldsymbol{i i}, \boldsymbol{a}\right) \mathbf{i i}, \boldsymbol{b}\right)$ of Theorem 2 , hence, $\tilde{v}$ is unanimous and candidate stable according to BDS2.

We have to remark that, apart from the case in which only three candidates are at stake, Theorem 1 and Theorem 2 are in the line of the results in Barberà et al. [3] in the study of strategy-proof social choice correspondences. Nevertheless, we want to highlight that strategyproofness and candidate stability are independent conditions. Basically, strategy-proofness (nonmanipulability via preferences) is a monotonicity condition on the preferences of the voters expressed in a fixed agenda framework, whereas candidate stability is defined in variable agenda settings, and implies consistency conditions in the choice at different ballots. The similarity of the results is due to the relation of both frameworks with some version of the Arrow's Theorem. In the following section we will show that the differences are not exclusive to the BDS2 domain.

In order to close this section, we should mention that we could obtain the same results in a

\footnotetext{
${ }^{9}$ The theorems admit other proofs. For instance, following Ehlers et al. [11], we can show that if a voting correspondence is candidate stable according BDS1 or BDS2 and unanimous, it is Pareto Efficient when the agenda contains at least $\# \mathcal{C}-1$ candidates. Then, from the results of Denicolò [9] on non-binary choice, we can develop the arguments that lead us to complete the proof. Denicolò's theorems are not concerned with candidate stability, but they make use of consistency conditions that can be derived from both BDS versions of candidate stability.
} 
more general set up in which voters are also endowed with preferences over sets of candidates. Hence, it would be possible to define voting rules that use voters' preferences over sets as inputs for the social choice, instead of their preferences over single candidates. Of course, these voting rules would operate in restricted domains, since voters' preferences would belong to the BDS domains. In this general framework, it is easy to see that Lemma 2 and Lemma 3 would still hold, since the results only depend on candidates' preferences over sets. So the main difficulty seems to be to obtain a rationalizability argument for candidate stable voting rules in a restricted domain of preferences. Nevertheless, for any unanimous and BDS (1 or 2) candidate stable voting procedure we can define a group decision function taking as reference the results of the social choice when only two candidates are relevant in the election. In such situations, the relevant information for the social choice mechanism is the same when voters report their preferences over set or they report their preferences over single candidates. ${ }^{10}$ This auxiliary group decision rule would be defined over voters' preferences over single candidates and these preferences are unrestricted. Thus, we could invoke again a version of Arrow's Theorem and apply to this general framework the same arguments we use in the proofs of Theorems 1 and 2 (up to minimal modifications) in order to obtain equivalent results. ${ }^{11}$ As the general framework does not provide additional insights in the problem, we have preferred to present the less general framework and we have kept the original DJL framework.

\section{Candidate Stability under Other Domains of Preferences over Sets}

The results stated in the previous section depend dramatically on how candidates are supposed to compare the sets of candidates. Both BDS scenarios are plausible when candidates can make use of plenty of information and construct preferences over sets in a somehow sophisticated way. Moreover, in both BDS scenarios the domains of admissible preferences over sets of candidates are very large. For each preference relation over candidates there are infinite utility functions

\footnotetext{
${ }^{10}$ If a voter $i$ prefers the candidate $a$ to the candidate $b$, for all consistent BDS preferences she must prefer the set $\{a\}$ to the set $\{a, b\}$ and the set $\{a, b\}$ to the set $\{b\}$.

${ }^{11} \mathrm{~A}$ proof is available from the author upon request.
} 
representing it, and many different orderings over $2^{\mathcal{C}} \backslash\{\varnothing\}$ can be devised from each preference ordering over candidates. Therefore, as we have seen, many possibilities of profitable withdrawal arise. In this section, we assume that candidates cannot assess any probability distribution on the set of elected candidates. In such situations, candidates may have extreme attitudes towards risk, consistent with the leximin, maximin or maximax preferences. ${ }^{12}$ They may also be reluctant to express strict preference among sets which are not clearly better or worse than other. In this case the candidates may use other criteria to construct their preferences over sets like, for example, the Gärdenfors preferences.

\section{Candidate Stability for Leximiners}

Leximin preferences reflect the way in which sophisticated pessimistic individuals construct their preferences over sets. When comparing two sets, a leximiner first compares the worst alternatives in each set. If they are the same, leximiners care about the next to the worst ones, and if they are again the same, leximiners follow the procedure in the same fashion. We express this formally:

Definition 6 (Leximin Preferences). Let $a \in \mathcal{C}, P_{a} \in \mathcal{P}^{a}$ and $X \in 2^{\mathcal{C}} \backslash\{\varnothing\}$. First define $X^{1}\left(P_{a}\right)=\operatorname{bottom}\left(X, P_{a}\right)$. Then proceed inductively. Once $X^{t}\left(P_{a}\right)$ is defined for any integer $t$ define $X^{t+1}\left(P_{a}\right)=\{\varnothing\}$ if $X=\cup_{i=1}^{t} X^{i}\left(P_{a}\right)$ and $X^{t+1}\left(P_{a}\right)=\operatorname{bottom}\left(\left[X \backslash \cup_{i=1}^{t} X^{i}\left(P_{a}\right)\right], P_{a}\right)$ otherwise.

The ordering $\succsim \in \mathcal{D}$ is the leximin preference over sets extending the preference ordering over candidates $P_{a}, \succsim=\succsim^{\text {lex }}\left(P_{a}\right)$, if and only if for all $X, Y \in 2^{\mathcal{C}} \backslash\{\varnothing\}$ :

$$
X \succ Y \Leftrightarrow X^{t^{\prime}}\left(P_{a}\right) P_{a} Y^{t^{\prime}}\left(P_{a}\right)
$$

where $t^{\prime}$ is the smallest integer $t$ such that $X^{t}\left(P_{a}\right) \neq Y^{t}\left(P_{a}\right)$.

Then, the domain of admissible leximin preferences can be defined by:

$$
\mathcal{D}_{a}^{\text {lex }}=\left\{\succsim \in \mathcal{D}, \text { such that there is } P_{a} \in \mathcal{P}^{a} \text { with } \succsim=\succsim^{\text {lex }}\left(P_{a}\right)\right\} .
$$

\footnotetext{
${ }^{12}$ These preference domains were first proposed in Pattanaik [18] and [19].
} 
Notice that for each $P_{a} \in \mathcal{P}^{a}$, there is only one leximin preference over sets $\left(\# \succsim^{\text {lex }}\left(P_{a}\right)=1\right)$. Moreover, as it is shown in Pattanaik [19], leximin preferences are incompatible with conditional expected utility maximization. These two facts may suggest that the opportunities of profitable withdrawal must be considerably reduced in the leximin scenario. The following lemma shows that this conjecture is not true.

Lemma 4. A voting correspondence $v$ is leximin candidate stable if and only if for all $a \in \mathcal{C}$, $P \in \mathcal{P}^{\mathcal{V}}:$

i) ("no-harm") if $a \in v(\mathcal{C}, P)$, then $v(\mathcal{C}, P) \subseteq(v(\mathcal{C} \backslash\{a\}, P) \cup\{a\})$;

ii) ("insignificance") if $a \notin v(\mathcal{C}, P)$, then $v(\mathcal{C}, P)=v(\mathcal{C} \backslash\{a\}, P)$.

Proof. Again, we start by proving sufficiency. Consider an arbitrary $P \in \mathcal{P}^{\mathcal{V}}$ and $a \in \mathcal{C}$ such that $a \in v(\mathcal{C}, P)$, then $v(\mathcal{C}, P) \subseteq(v(\mathcal{C} \backslash\{a\}, P) \cup\{a\})$. In order to simplify the notation, denote the sets $v(\mathcal{C}, P)=X$ and $v(\mathcal{C} \backslash\{a\}, P)=Y$. Consider an arbitrary $P_{a} \in \mathcal{P}^{a}$ and find the smallest integer $t^{\prime}$ such that $X^{t^{\prime}}\left(P_{a}\right) \neq Y^{t^{\prime}}\left(P_{a}\right)$. Notice that $X^{t^{\prime}}\left(P_{a}\right) \in Y$, and thus for some integer $t^{\prime \prime}>t^{\prime}$, $X^{t^{\prime}}\left(P_{a}\right)=Y^{t^{\prime \prime}}\left(P_{a}\right)$. This implies that $X^{t^{\prime}}\left(P_{a}\right) P_{a} Y^{t^{\prime}}\left(P_{a}\right)$, and $v(\mathcal{C}, P) \succ^{l e x}\left(P_{a}\right) v(\mathcal{C} \backslash\{a\}, P)$. As the choice of $P_{a}$ was arbitrary, this implies that for all $\succsim \in \mathcal{D}_{a}^{l e x}, v(\mathcal{C}, P) \succ v(\mathcal{C} \backslash\{a\}, P)$. Then, elected candidates do not have incentives to quit. Now suppose that $a \notin v(\mathcal{C}, P), v(\mathcal{C}, P)=$ $v(\mathcal{C} \backslash\{a\}, P)$. Since $a$ 's withdrawal has no effect on the social choice, she can not improve by leaving. This concludes the proof of sufficiency, since the choice of $P$ was arbitrary.

Now we prove necessity. First, let us prove item i). Assume $v$ is leximin candidate stable but there are $a, b \in \mathcal{C}, P \in \mathcal{P}^{\mathcal{V}}$ such that $a, b \in v(\mathcal{C}, P)$ and $b \notin v(\mathcal{C} \backslash\{a\}, P)$. We can find a preference ordering $P_{a}^{\prime} \in \mathcal{P}^{a}$ such that $b=\operatorname{bottom}\left(\mathcal{C}, P_{a}^{\prime}\right)$, which implies that $v(\mathcal{C} \backslash\{a\}, P) \succ^{l e x}\left(P_{a}^{\prime}\right) v(\mathcal{C}, P)$ and contradicts leximin candidate stability. On the other hand, assume $v$ is candidate stable, but ii) does not hold. Hence, there are $a \in \mathcal{C}$ and $P \in \mathcal{P}^{\mathcal{V}}$, such that $a \notin v(\mathcal{C}, P)$ but $v(\mathcal{C}, P) \neq v(\mathcal{C} \backslash\{a\}, P)$. Now find $P_{a}^{\prime \prime} \in \mathcal{P}^{a}$ such that for all $b \in(v(\mathcal{C} \backslash\{a\}, P) \backslash v(\mathcal{C}, P)), c \in(v(\mathcal{C}, P) \cap v(\mathcal{C} \backslash\{a\}, P))$ and $d \in(v(\mathcal{C}, P) \backslash v(\mathcal{C} \backslash\{a\}, P))$, $b P_{a}^{\prime \prime} c P_{a}^{\prime \prime} d$. Notice that $v(\mathcal{C} \backslash\{a\}, P) \succ^{l e x}\left(P_{a}^{\prime \prime}\right) v(\mathcal{C}, P)$, which again contradicts leximin candidate stability. 
Lemma 4 shows that leximin preferences also introduce strong restrictions on candidate stable voting correspondences. The implications are very similar to those derived for the conditional expected utility scenarios. Namely, the withdrawal of an elected candidate cannot harm the remaining elected candidates (we have dubbed this property as no-harm), while the exit of a non-elected candidate cannot affect the final outcome (insignificance). Albeit the leximin domain reduces the strategic incentives to withdraw from the ballot, only rules endowing some voters with veto power satisfy unanimity, no-harm, and insignificance.

\section{Theorem 3.}

i) If a voting correspondence $v$ is unanimous and leximin candidate stable then there is a subset of voters $S \subseteq \mathcal{V}$ such that for all $A \in\left\{\mathcal{C},\{\mathcal{C} \backslash\{a\}\}_{a \in \mathcal{C}}\right\}$ and for all $P \in \mathcal{P}^{\mathcal{V}}$ :

a) $v(A, P) \subseteq$ Pareto $\left(A, P_{S}\right)$,

b) for any $a \in \mathcal{C}, v(A, P) \neq\{a\}$ if there are $i \in S$ and $b \in A$ with $b P_{i} a$,

c) for any $a \in \mathcal{C}, \cup_{i \in S} \operatorname{top}\left(\mathcal{C} \backslash\{a\}, P_{i}\right) \subseteq v(\mathcal{C} \backslash\{a\}, P)$.

ii) Let $v$ be such that for all $A \in 2^{\mathcal{C}} \backslash\{\varnothing\}$ and all $P \in \mathcal{P}^{\mathcal{V}}, v(A, P)=\operatorname{Pareto}(A, P)$, then $v$ is unanimous and leximin candidate stable.

Proof. Item i) is an immediate corollary to Theorem 4 and it is proved in the Appendix 1. We prove item ii). It is clear that Pareto correspondences are unanimous. On the other hand, consider an arbitrary profile of voters preferences $P \in \mathcal{P}^{\mathcal{V}}$ and some $a \in \mathcal{C}$. Assume first that $a \in \operatorname{Pareto}\left(\mathcal{C}, P_{S}\right)$ and take an arbitrary $b \in \operatorname{Pareto}(\mathcal{C}, P)$. Notice that if $b$ is not dominated by any alternative in $\mathcal{C}$, it cannot be dominated by any alternative in $\mathcal{C} \backslash\{a\}$. Then, $b \in \operatorname{Pareto}(\mathcal{C} \backslash\{a\}, P)$, and for all $a \in \mathcal{C}, \operatorname{Pareto}(\mathcal{C}, P) \subseteq($ Pareto $(\mathcal{C} \backslash\{a\}, P) \cup\{a\})$. Moreover, those candidates who were dominated when $a$ was at stake, were dominated by some candidate in $\mathcal{C} \backslash\{a\}$. Notice also that the candidates that are not Pareto dominated when all candidates are at stake are not dominated by any alternative in $\mathcal{C} \backslash\{a\}$. Hence, whenever $a \notin v(\mathcal{C}, P)$, Pareto $\left(\mathcal{C}, P_{S}\right)=$ Pareto $\left(\mathcal{C} \backslash\{a\}, P_{S}\right)$. This suffices to prove that the Pareto correspondence satisfies the requirements of Lemma 4. 
As any unanimous and leximin candidate stable voting correspondence never selects Pareto dominated candidates, Theorem 3 can be viewed as a positive result: many interesting voting correspondences do not provide incentives for candidates to withdraw if they use leximin preferences to compare sets of candidates. ${ }^{13}$ The reader can check that the conditions in item i) of Theorem 3 are also sufficient when there are only three candidates, but they are not when there are more than four candidates. We want remark that, besides Pareto correspondences, other rules satisfy the requirements of Theorem 3 independently of the size of the set of candidates. These points are illustrated in the following examples.

Example 2. Let $\mathcal{V}=\{1,2,3,4\}$ and $\mathcal{C}=\{a, b, c, d\}$, and define the voting correspondence $v^{\prime}$ in the following way. For any $A \in 2^{\mathcal{C}} \backslash\{\varnothing\}$ and any $P \in \mathcal{P}^{\mathcal{V}}$ :

$$
v^{\prime}(A, P)= \begin{cases}\cup_{i=1}^{2} \operatorname{top}\left(A, P_{i}\right) & \text { if } A=\mathcal{C}, \\ \cup_{i=1}^{3} \operatorname{top}\left(A, P_{i}\right) & \text { if } A \varsubsetneqq \mathcal{C} .\end{cases}
$$

The voting correspondence $v^{\prime}$ satisfies item i) of Theorem 3 but it is not leximin candidate stable. Consider the preference profile $P$, such that $a P_{1} b P_{1} c P_{1} d, b P_{2} c P_{2} d P_{2} a$ and $c P_{3} d P_{3} a P_{3} b$. Notice that $v^{\prime}(\mathcal{C}, P)=\{a, b\}$, while $v^{\prime}(\mathcal{C} \backslash\{c\}, P)=\{a, b, d\}$ which violates insignificance.

Conside now the voting correspondence $v^{\prime \prime}$ defined for all $A \in 2^{\mathcal{C}} \backslash\{\varnothing\}$ and all $P \in \mathcal{P}^{\mathcal{V}}$ as follows:

$$
v^{\prime \prime}(A, P)= \begin{cases}\mathcal{C} \backslash\left\{\operatorname{top}\left(\mathcal{C}, P_{4}\right)\right\} & \text { if } \cup_{i=1}^{4} \operatorname{top}\left(A, P_{i}\right)=\mathcal{C} \\ \cup_{i=1}^{4} \operatorname{top}\left(A, P_{i}\right) & \text { otherwise. }\end{cases}
$$

The reader can easily check that the voting correspondence $v^{\prime \prime}$ is unanimous and leximin candidate stable.

The remainder of this section is devoted to presenting the possibilities that arise when the domains of candidates' preferences over sets are even more restricted. In particular, their preferences could be consistent with maximin, maximax or Gärdenfors preferences. In these scenarios, candidate stability is not very stringent, and many interesting rules are not affected

\footnotetext{
${ }^{13}$ Campbell et al. [6] prove that a social choice correspondence is unanimous and strategy-proof according to leximin if and only if it is the union of the best alternatives of an arbitrary group of voters at any preference profile.
} 
by the incentives of the candidates to quit the election. On the other hand, the arguments we use in the proofs of the previous theorems do not apply to these new environments. The crucial point is that candidate stability becomes so weak that it is not possible to construct well-defined auxiliary group decision rules. Morevoer, we cannot employ the classical results on quasitransitive rationalizability of voting correspondences that we have used in the proofs of the previous theorems. Some weaker rationalizability result can be obtained at the cost of introducing other additional conditions apart from unanimity and candidate stability. But in any case, no clear characterization would be available. However, these partial results provide interesting hints about the voting correspondences that satisfy these mild versions of candidate stability. Therefore, instead of looking for characterization results our strategy will be the following. First, we derive the specific implications of candidate stability in each context. Then we will provide some relevant examples of voting correspondences that are candidate stable in these new environments.

\section{Candidate Stability for Maximiners}

The maximin preferences can be interpreted as the behavior of extreme risk averters who only care about the worst alternative in a set.

Definition 7. The ordering $\succsim \in \mathcal{D}$ is the maximin ordering over sets extending the preference ordering over candidates $P_{a} \in \mathcal{P}^{a}, \succsim=\succsim^{\min }\left(P_{a}\right)$, if and only if for all $X, Y \in 2^{\mathcal{C}} \backslash\{\varnothing\}$,

$$
X \succsim Y \Leftrightarrow \operatorname{bottom}\left(X, P_{a}\right) R_{a} \text { bottom }\left(Y, P_{a}\right)
$$

The domain $\mathcal{D}_{a}^{m i n}$ contains all the maximin orderings admissible for candidate a.

The implications of candidates stability when candidates are maximiners are much weaker than under leximin preferences. Notice that leximin preferences apply the maximin logic of comparisons of the worst elements in each set iteratively. Thus, whenever a maximiner finds profitable to quit an election, she would also improve in the case she were a leximiner. In Proposition 1, we specify the constrains that candidate stability introduces in the maximin scenario. We also present the family of core correspondences that satisfy unanimity and maximin candidate stability. 
The Pareto correspondence is an example of a core correspondence. A candidate $b$ is not selected by the Pareto correspondence if there is another candidate $a$ such that all the voters prefer $a$ to $b$. In this case, we say that the candidate $a$ blocks the selection of the candidate $b$ since all the voters prefer $a$ to $b$ and they have the power to enforce the social outcome to be $a$. Hence, we can say that when a society uses the Pareto correspondence as a voting correspondence, only the whole society has blocking power.

The distribution of the blocking power that is implicit in the definition of the Pareto correspondence is rather restrictive. We can think of a Parliament or a society in which the agreement of two thirds of its members or any other qualified majority rule is needed to impose an alternative. Core correspondences allow for general distributions of the blocking power. In order to describe these possibilities, we introduce the definition of Effectivity functions and core correspondences.

An Effectivity function $E$ is a mapping from the set of non-empty sets of voters, $2^{\mathcal{V}} \backslash\{\varnothing\}$, to $2^{\mathcal{C}} \backslash\{\varnothing\}$ such that $(i)$ for any $A \in 2^{\mathcal{C}} \backslash\{\varnothing\}, A \in E(\mathcal{V})$; and $(i i)$, for any $S \in 2^{\mathcal{V}} \backslash \varnothing, \mathcal{C} \in E(S)$. Given the effectivity function $E$, we say that the coalition $S \subseteq \mathcal{V}$ has the power to force the social outcome to belong to the set $A \subseteq \mathcal{C}$ if and only if $A \in E(S)$.

The coalition $S \subseteq \mathcal{V}$ blocks candidate $b$ at profile $P \in \mathcal{P}^{\mathcal{V}}$ if there is another candidate $a \in \mathcal{C}$ such that for all $i \in S, a P_{i} b$, and $a \in E(S)$, we say $a$ dominates $b$ at profile $P$. A coalition blocks a candidate if the coalition has the effective power to force the social outcome to be another candidate, and all the members of the coalition prefer this available candidate to the first one. For any $A \in 2^{\mathcal{C}} \backslash\{\varnothing\}$ and $P \in \mathcal{P}^{\mathcal{V}}$, the Core correspondence associated to the effectivity function $E, C_{E}$, selects the set of candidates in $A$ which are not dominated by any other candidate in $A$ at profile $P$.

\section{Proposition 1.}

i) A voting correspondence $v$ is maximin candidate stable if and only if for all $a \in \mathcal{C}$ and for all $P \in \mathcal{P}^{\mathcal{V}}, v(\mathcal{C}, P) \subseteq(v(\mathcal{C} \backslash\{a\}, P) \cup\{a\})$.

ii) Given an effectivity function $E$, if for all $A \in\left\{\mathcal{C},\{\mathcal{C} \backslash\{a\}\}_{a \in \mathcal{C}}\right\}$ and all $P \in \mathcal{P}^{\mathcal{V}}$ its associated Core correspondence, $C_{E}$, is non-empty, then $C_{E}$ is unanimous and maximin candidate 
stable.

Proof. i) In order to prove sufficiency, consider an arbitrary candidate $a \in \mathcal{C}$ and an arbitrary preference profile $P \in \mathcal{P}^{\mathcal{V}}$. Notice that $v(\mathcal{C}, P) \subseteq(v(\mathcal{C} \backslash\{a\}, P) \cup\{a\})$ implies that for all $P_{a} \in \mathcal{P}^{a}$, $\operatorname{bottom}\left(v\left(\mathcal{C}, P, P_{a}\right) \in v(\mathcal{C} \backslash\{a\}, P), P_{a}\right)$, and also bottom $\left(v(\mathcal{C}, P), P_{a}\right) R_{a} \operatorname{bottom}\left(v(\mathcal{C} \backslash\{a\}, P), P_{a}\right)$. As the choice of $a$ and $P$ was arbitrary this suffices to prove that no maximiner candidate has incentive to leave the ballot.

On the other hand, in order to prove necessity assume $v$ is maximin candidate stable but there are $a, b \in \mathcal{C}, P \in \mathcal{P}^{\mathcal{V}}$, such that $b \in v(\mathcal{C}, P), b \notin v(\mathcal{C} \backslash\{a\}, P)$. Take a preference $P_{a} \in \mathcal{P}^{a}$ such that $b=\operatorname{bottom}(\mathcal{C}, P)$, then we can find $\succsim \in \mathcal{D}_{a}^{\text {min }}$ such that $v(\mathcal{C} \backslash\{a\}, P) \succ v(\mathcal{C}, P)$ contradicting maximin candidate stability.

ii) First notice that the core correspondences are unanimous, since all the sets $A \in 2^{\mathcal{C}} \backslash\{\varnothing\}$ belong to $E(\mathcal{V})$. To check maximin candidate unanimity is also immediate. Just notice that if a candidate $a$ is not blocked when all the candidates are at stake, she cannot be blocked by a running candidate when a candidate leaves the fray. Then for all $a \in \mathcal{C}$ and all $P \in \mathcal{P}^{\mathcal{V}}$, $C_{E}(\mathcal{C}, P) \subseteq C_{E}(\mathcal{C} \backslash\{a\}, P)$

Item i) of Proposition 1 proves that maximin candidate stability only prevents the voting correspondence from shrinking the chosen set when a candidate withdraws. In item ii), we show that as maximin preferences reduce the candidates' incentives to withdraw, general distributions of the blocking power are admitted. In the following example, we present a unanimous and maximin candidate stable voting correspondence in which a simple (strict) majority of the voters is enough to block the election of a candidate.

Example 3. Consider a society with four voters and three candidates. For any $A \in 2^{\mathcal{C}} \backslash\{\varnothing\}$ and any $P \in \mathcal{P}^{\mathcal{V}}, \bar{v}(A, P)=\{a \in A$ s.t. for no $b \in A$ there are three voters preferring $b$ to $a\}$. The reader can check that $\bar{v}$ satisfies item i) of Proposition 1, and hence it is maximin candidate stable. ${ }^{14}$

\footnotetext{
${ }^{14} \mathrm{We}$ want to remark that this example cannot be generalized to arbitrary sizes of the sets of voters and candidates. The effectivity function for which any strict majority of the voters can block a candidate may generate empty-cores at some profiles of preferences.
} 


\section{Candidate Stability and Gärdenfors Preferences}

Gärdenfors preferences match scenarios in which the candidates are not extreme risk averters, but they are very reluctant to express strict preference between sets unless one set clearly improves the other one. These preferences can be suitable when the candidates have very little information on the resolution of the social choice.

Definition 8. The ordering $\succsim \in \mathcal{D}$ is the Gärdenfors ordering over sets extending the preference ordering over candidates $P_{a}, \succsim=\succsim^{G}\left(P_{a}\right)$, if and only if for all $X, Y \in 2^{\mathcal{C}} \backslash\{\varnothing\}$,

$$
X \succsim Y \Leftrightarrow \text { for all } b \in X \backslash Y, c \in X \cap Y \text { and } d \in Y \backslash X, b P_{a} c P_{a} d \text {. }
$$

The domain $\mathcal{D}_{a}^{G}$ contains all the Gärdenfors orderings admissible for candidate a.

Gärdenfors preferences can be equivalently defined in the following way. A candidate $a$ with preferences over candidates $P_{a}$, prefers the set $X$ to the set $Y$, if for some $u_{a}$ fitting $P_{a}$, for all possible prior assessments the conditional expected utility obtained from the set $X$ is always higher than the conditional expected utility that the set $Y$ yields. ${ }^{15}$ Gärdenfors preferences fit uncertain environments in which any distribution of probabilities among alternatives is plausible and candidates are reluctant to use a specific one.

It is clear that the Gärdenfors preferences are weak, in the sense that it is difficult to obtain strict preference over sets. This fact reduces the incentives to quit of the candidates. In fact, we will see that even rules based on majority comparison are Gärdenfors candidate stable.

Let $\mathcal{B}$ denote the set of complete binary relations on $\mathcal{C}$. For any $P \in \mathcal{P}^{\mathcal{V}}$, the strict majority

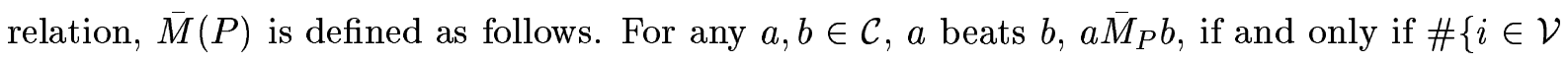
s.t. $\left.a P_{i} b\right\}>\#\left\{j \in \mathcal{V}\right.$ s.t. $\left.b P_{i} a\right\}$. The weak component of the majority relation, $M(P)$, is defined in the usual way. For any $A \in 2^{\mathcal{C}} \backslash\{\varnothing\}$ and for all $P \in \mathcal{P}^{\mathcal{V}}$, the top cycle of the majority relation $t c(A, P)$ is defined as the minimal (w.r.t. inclusion) set on $A$ such that all its elements defeat all the candidates who belong to $A \backslash t c(A, P)$ by majority comparisons. ${ }^{16}$ The top cycle

\footnotetext{
${ }^{15} \mathrm{~A}$ proof of this statement can be found in Ching and Zhou [7]. They make use of degenerate lotteries but a similar argument applies to our scenario.

${ }^{16}$ Analogously, we could define the Top Cycle of any binary relation $B \in \mathcal{B}$ in the same fashion. The results in the item ii) of Proposition 2 are also valid for the Top Cycle of any binary relation $B \in \mathcal{B}$.
} 
correspondence is never empty and satisfies interesting properties. For instance, it is a singleton if and only if there is a candidate who beats all the remaining ones by majority comparison, that is the top cycle equals the Condorcet winner whenever the latter exists.

\section{Proposition 2.}

i) A voting correspondence $v$ is Gärdenfors candidate stable if and only if for all $a \in \mathcal{C}$ and all $P \in \mathcal{P}^{\mathcal{V}} a \notin v(\mathcal{C}, P)$ implies $v(\mathcal{C}, P)=v(\mathcal{C} \backslash\{a\}, P)$.

ii) The majority Top Cycle Correspondence is unanimous and Gärdenfors candidate stable.

Proof. i) In order to prove sufficiency, consider an arbitrary preference profile $P \in \mathcal{P}^{\mathcal{V}}$ and a candidate $a \in \mathcal{C}$. Notice first that if $a \in v(\mathcal{C}, P)$, the self preference of the candidates implies that $v(\mathcal{C}, P) \succsim v(\mathcal{C} \backslash\{a\}, P)$ for all $\succsim \in \mathcal{D}_{a}^{G}$. On the other hand, if $a \notin v(\mathcal{C}, P)$ she cannot improve by withdrawing, since she cannot change the outcome of the election.

Finally, let us check necessity. Assume there is $P \in \mathcal{P}^{\mathcal{V}}$, such that a non-elected candidate $a \notin v(\mathcal{C}, P)$ can affect the result of social choice and $v(\mathcal{C}, P) \neq v(\mathcal{C} \backslash\{a\}, P)$. Then, as $a \notin$ $v(\mathcal{C}, P) \cup v(\mathcal{C} \backslash\{a\}, P)$, we can find $P_{a} \in \mathcal{P}^{a}$ such that for all $b \in v(\mathcal{C} \backslash\{a\}, P) \backslash v(\mathcal{C}, P), c \in$ $v(\mathcal{C}, P) \cup v(\mathcal{C} \backslash\{a\}, P)$ and $d \in v(\mathcal{C}, P) \backslash v(\mathcal{C} \backslash\{a\}, P), b P_{a} c P_{a} d$. But this implies that there is $\succsim \in \mathcal{D}_{a}^{G}$ such that $v(\mathcal{C} \backslash\{a\}, P) \succ v(\mathcal{C}, P)$, which contradicts Gärdenfors candidate stability.

ii) Unanimity of the top cycle correspondence is clear. To prove Gärdenfors candidate stability is also immediate. If a candidate $a \notin t c(\mathcal{C}, P)$ leaves the stake then all candidates within $t c(\mathcal{C}, P)$ beat all candidates outside it. Since $t c(\mathcal{C}, P)$ is minimal for $\mathcal{C}$, it is also minimal for $\mathcal{C} \backslash\{a\}$. Thus, for all $P \in \mathcal{P}^{\mathcal{V}}$ and for all $a \notin t c(\mathcal{C}, P), t c(\mathcal{C}, P)=t c(\mathcal{C} \backslash\{a\}, P)$.

In item i) of Proposition 2, we have proved that under Gärdenfors preferences the incentives to leave the ballot only affect the non-elected candidates. In fact, candidate stability reduces to insignificance of the non-elected candidates and it is equivalent to the DJL's formulation of strong candidate stability in single-valued elections. Nevertheless, the implications of insignificance are dramatically different in multivalued environments. In the single-valued case, it is equivalent to the Arrow's Choice Axiom and it implies the transitive rationalizability of the voting procedure. 
However, as item ii) shows, its implications are much weaker when applied to multivalued environments.

\section{Candidate Stability for Maximaxers}

Finally, maximax extension is obviously defined as the way in which extremely optimistic people compare sets of candidates.

Definition 9. The ordering $\succsim \in \mathcal{D}$ is the maximax ordering over sets extending the preference ordering over candidates $P_{a}, \succsim=\succsim^{\text {max }}\left(P_{a}\right)$, if and only if for all $X, Y \in 2^{\mathcal{C}} \backslash\{\varnothing\}$,

$$
X \succsim Y \Leftrightarrow \operatorname{top}\left(X, P_{a}\right) R_{a} \operatorname{top}\left(Y, P_{a}\right)
$$

The domain $\mathcal{D}_{a}^{\max }$ contains all the maximax orderings admissible for candidate a.

Although the maximax and Gärdenfors domains are independent, the maximax version of candidate stability is even weaker than the Gärdenfors' one. We directly introduce another example of correspondence that fits the requirements of maximax candidate stability, the Uncovered Set. ${ }^{17}$ Let $\# \mathcal{V}$ be odd. We say $a$ is covered by $b$ according to the majority relation $M(P)$ if $b \bar{M}(P) a$ and for all $c$ such that $a \bar{M}(P) c$, also $b \bar{M}_{P} c$. For all $A \subseteq \mathcal{C}$, and for all $P \in \mathcal{P}^{\mathcal{V}}$ define the Uncovered Set of the majority relation $u c(A, P)$, as the set of candidates in $A$ who are not covered by any other candidate in $A$.

\section{Proposition 3.}

i) A voting correspondence $v$ is candidate stable according to the maximax extension if and only if for all $a \in \mathcal{C}$ and all $P \in \mathcal{P}^{\mathcal{V}}, a \notin v(\mathcal{C}, P)$, implies $v(\mathcal{C} \backslash\{a\}, P) \subseteq v(\mathcal{C}, P)$.

ii) If \#V is odd, the Uncovered Set of the majority relation is unanimous and maximax candidate stable.

Proof. i) In order to prove sufficiency, consider an arbitrary profile of voters preferences $P \in$ $\mathcal{P}^{\mathcal{V}}$. Notice first that an elected candidate never has incentives to leave the fray, since for all $P_{a} \in \mathcal{P}^{a}, a=\operatorname{top}\left(v(\mathcal{C}, P) P_{a}\right) P_{a} \operatorname{top}\left(v(\mathcal{C} \backslash\{a\}, P), P_{a}\right)$. Moreover, if for all $a \notin v(\mathcal{C}, P)$,

\footnotetext{
${ }^{17}$ See Miller [15] and Chapter 9 in Moulin [16].
} 
$v(\mathcal{C} \backslash\{a\}, P) \subseteq v(\mathcal{C}, P), \operatorname{top}\left(v(\mathcal{C} \backslash\{a\}, P), P_{a}\right) \subseteq v(\mathcal{C}, P)$. This implies that for all $\succsim \in \mathcal{D}_{a}^{\text {max }}$, $v(\mathcal{C}, P) \succsim v(\mathcal{C} \backslash\{a\}, P)$. Hence, sufficiency holds.

Necessity also follows immediately. Assume ad contrarium that $v$ is maximax candidate stable but there are $P \in \mathcal{P}^{\mathcal{V}}$ and $a \notin v(\mathcal{C}, P)$ such that there is another candidate $b \in$ $v(\mathcal{C} \backslash\{a\}, P) \backslash v(\mathcal{C}, P)$. Take $P_{a} \in \mathcal{P}^{a}$, such that $b=\operatorname{top}\left(\mathcal{C} \backslash a, P_{a}\right)$, as $a \notin v(\mathcal{C}, P)$. This suffices to ensure the existence of a maximax preference over sets $\succsim \in \mathcal{D}_{a}^{\max }$ such that $v(\mathcal{C} \backslash\{a\}, P) \succ$ $v(\mathcal{C}, P)$, which contradicts candidate stability.

ii) Notice that the uncovered set is unanimous since at unanimous profiles there is a candidate that beats all the remaining candidates. Assume that the uncovered set is not maximax candidate stable. Then, there are a preference profile $P \in \mathcal{P}^{\mathcal{V}}$, and two candidates $a, b \in \mathcal{C}$ such that $a \notin u c(\mathcal{C}, P)$ and $b \in u c(\mathcal{C} \backslash\{a\}, P)$, but $b \notin u c(\mathcal{C}, P)$. Notice that if $b$ is not covered by any $c \in \mathcal{C} \backslash\{a\}$ but it is covered by some candidate in $\mathcal{C}$, then $b$ is covered by $a$. By the transitivity of the covering relation and as $a \notin u c(\mathcal{C}, P), b$ also should be covered by some candidate in $\mathcal{C} \backslash\{a\}$, a contradiction. This implies that the uncovered set satisfies the conditions of item i) and it is always maximax candidate stable.

Maximax candidate stability is slightly weaker than Gärdenfors' candidate stability. Hence, any Gärdenfors candidate stable voting correspondence is also maximax candidate stable, while the converse implication does not hold. In fact, it is easy to see that the uncovered set of the majority relation is not candidate stable under Gärdenfors preferences.

Example 4. Let $\mathcal{V}=\{1,2,3\}, \mathcal{C}=\{a, b, c, d, e\}$. Consider the preference profile $P \in \mathcal{P}^{\mathcal{V}}$ such that $a P_{1} e P_{1} b P_{1} c P_{1} d ; b P_{2} c P_{2} d P_{2} a P_{2} e$ and $c P_{3} e P_{3} d P_{3} a P_{3} b$, then $u c(\mathcal{C}, P)=\{a, b, c, e\}$, whereas $u c(\mathcal{C} \backslash d, P)=\{a, b, c\}$. If the candidate $d^{\prime} s$ preferences over candidates are $d P_{j} a P_{j} b P_{j} c P_{j}$ e and she uses the Gärdenfors criterion to compare sets of candidates, $d$ has an incentive to quit.

In order to close this section a few remarks are in order. First, we have seen that Condorcet consistent rules as the Top Cycle and the Uncovered Set satisfy maximax candidate stability. However, we have not mentioned the effects of the incentives to withdraw for the candidates at other prominent class of voting rules, the family of scoring methods. Under scoring methods, each voter assigns points to each candidate, linearly increasing with her ranking in the voter's 
preferences. The candidates who accumulate more points are finally elected. It is not difficult to check that scoring rules are not candidate stable neither under the maximin domain nor under maximax preferences.

Finally note that a voting correspondence $v$ is maximin and Gärdenfors (or maximax) candidate stable if and only if $v$ is candidate stable according to the leximin extension. This final remark implies that a core correspondence (that is unanimous and maximin candidate stable) is maximax candidate stable only if it is the Pareto correspondence. ${ }^{18}$

\section{Overlap between Voters and Candidates}

We have already discussed that our assumption on non-voting candidates is suitable in many voting situations and can be a good approximation to large elections. However, the possibility of being at the same time a voter and a candidate should not be ruled out and it deserves interest on its own. Unfortunately, it is not trivial to extend the analysis of strategic incentives of the candidates to this environment.

A first difficulty on the analysis of environments with voting candidates is derived from the restriction on the self-preference of the candidates. If a candidate can vote, the voting correspondence no longer operates in an universal domain. Hence, we cannot use directly any version of the Arrow's Theorem. Moreover, unanimity becomes empty of content. Whenever there are at least two voting candidates, no unanimous profile exists. This problem can be overcome by introducing stronger versions of unanimity. For instance, we say a voting correspondence is strongly unanimous if whenever there is a running candidate who is the best candidate at stake for all the voters who are not candidates, and the second best for all the voting candidates, the voting correspondence chooses this candidate. Formal definition follows.

We say $B \subseteq \mathcal{C}$ is a restricted top-set of profile $P$ if for all $i \in \mathcal{V}$, for all $b \in B, c \in(\mathcal{C} \backslash\{i\}) \backslash B$, $b P_{i} c$. We write $P \in \mathcal{P}^{\mathcal{V}}(B)$.

\footnotetext{
${ }^{18}$ Demange [8] shows that many core correspondences, those generated from convex effectivity functions, are not manipulable according to the maximax criterion. This family of core correspondences strictly includes Pareto correspondences. Hence, we cannot state that candidate stability is generically weaker than strategy-proofness, as many maximax strategy-proof social choice correspondences are not maximax candidate stable.
} 
Definition 10. The voting correspondence $v$ is strongly unanimous if for all $P \in \mathcal{P}^{\mathcal{V}}$, and all $B \subseteq \mathcal{C}$, whenever there is $b \in B$ such that $b$ is a restricted top-set of $P$ then $v(B, P)=b$.

The above problems put in jeopardy our normative analysis but are not overwhelming. However, the most important difficulty arises when defining the implications of candidate stability. If candidates can vote, our Lemmata 2, 3, 4 and the items i) of Propositions 1, 2, 3 do not apply, since the social choice depends on the preferences of the withdrawing candidates. Notice that the society could use punishment strategies to eliminate the incentives of the candidates to leave. For instance, we can think of a voting correspondence which selects the worst candidate according to the preferences of the withdrawing candidate. Of course, such choices may be incompatible with strong unanimity, but other interesting examples can be devised.

Example 5. Consider a society in which all the voters are also candidates, $\mathcal{C}=\mathcal{V}$. Define the restricted Pareto correspondence in the following way. For all $A \in 2^{\mathcal{C}} \backslash\{\varnothing\}$ and all $P \in \mathcal{P}^{\mathcal{V}}$ :

$$
\operatorname{Pareto}^{*}(A, P)=\left\{a \in A \text { s.t. for no } b \in A, b P_{i} a, \text { for all } i \in(\mathcal{V} \backslash\{a\})\right\} \text {. }
$$

Now define the voting correspondence $v^{*}$ in the following way. For all $P \in \mathcal{P}^{\mathcal{V}}$ and all $a \in \mathcal{C}$,

$$
\begin{gathered}
v^{*}(\mathcal{C}, P)=\text { Pareto }^{*}(\mathcal{C}, P), \text { and } \\
v^{*}(\mathcal{C} \backslash\{a\}, P)=\operatorname{bottom}\left(\text { Pareto }^{*}(\mathcal{C} \backslash\{a\}, P), P_{a}\right) .
\end{gathered}
$$

It is immediate to check that $v^{*}$ is candidate stable with respect to the domains of preferences over sets we have presented. Notice that Pareto* $(\mathcal{C}, P) \subseteq$ Pareto $^{*}(\mathcal{C} \backslash\{a\}, P)$. Hence for any $P_{a}$ and for all $b \in \operatorname{Pareto}^{*}(\mathcal{C}, P), b R_{a} v(\mathcal{C} \backslash\{a\}, P)$. Therefore, the outcome after the withdrawal cannot improve the result when all the candidates run the election. Moreover, $v^{*}$ is also strongly unanimous. However, the use of the punishment could imply that the social choice may be against the interest of almost all the members of the society when $\# \mathcal{V}$ is very large.

A possible approach consists of introducing some candidate stability condition without modeling explicitly candidates' incentives to abandon the election. This possibility is proposed in Ehlers et al. [11] and Eraslan et al. [12], where a strong candidate stability condition is provided. We can weaken their condition and ask for voting correspondences satisfying "no-harm" 
of the withdrawal of an elected candidate and "insignificance" of non-elected candidates. ${ }^{19}$ In this case we can obtain a result similar to the item i) of Theorem 3 .

Theorem 4. If a voting correspondence $v$ satisfies strong unanimity, no-harm and insignificance, then there is a set of voters $S \subseteq(\mathcal{V} \backslash \mathcal{C}$ ) (who are not candidates) such that, for all $P \in \mathcal{P}^{\mathcal{V}}$ :

a) $v(A, P) \subseteq \operatorname{Pareto}\left(A, P_{S}\right)$,

b) for any $a \in A, v(A, P) \neq\{a\}$ if there are $i \in S$ and $b \in A$ with $b P_{i} a$, and

c) for any $a \in \mathcal{C}, \cup_{i \in S} \operatorname{top}\left(\mathcal{C} \backslash\{a\}, P_{i}\right) \subseteq v(\mathcal{C} \backslash\{a\}, P)$.

The above theorem has a chief drawback. By imposing an "ad hoc" stability condition, it does not consider the real incentives of the candidates to withdraw. As the general analysis of the voting candidates case seems out of reach, DJL have proposed the study of specific classes of voting rules in single-valued environments. In their Theorem 2 , they have proved that candidate stability is incompatible with a strong version of unanimity (dubbed m-2 unanimity) for a large family of voting procedures satisfying a restricted version of monotonicity (that they called top-pair monotonicity). ${ }^{20}$ The reader can check that the arguments in the proof of Theorem 2 in DJL can be applied with minimal modifications to multivalued environments when candidates preferences over sets belong to the conditional expected utility and leximin domains. Hence, we can state that there is no voting correspondence satisfying $m-2$ unanimity, top-pair monotonicity and either BDS1 candidate stability, BDS2 candidate stability or leximin candidate stability. ${ }^{21}$

\footnotetext{
${ }^{19}$ See Lemma 4.

${ }^{20} \mathrm{~A}$ voting rule satisfies $m-2$ unanimity if whenever there are a group of $\# \mathcal{V}-2$ voters who prefer a candidate above the remaining ones, that candidate is elected. A voting rule is top-pair monotonic if i) whenever there are two candidates who are a top-set, then the remaining candidates are not chosen, and ii) an increase of the support of a candidate never harms.

${ }^{21} \mathrm{~A}$ proof of this statement is available from the author upon request.
} 


\section{Appendices}

\subsection{Appendix 1: Proofs of the Theorems}

The proofs below do not appear in the same order as the results. This economizes the exposition, as some results that appear later in the paper (namely, Theorem 4) are used in the proofs of ones stated earlier (Theorems 1, 2 and 3). We have preferred to highlight the less general theorems regarding the non-overlap between voters and candidates' sets because they admit much better interpretation in terms of candidates incentives.

We have already mentioned that the main theorem exploits the relation between candidate stable voting correspondences and rationalizable group decision rules. This relation permits us to employ at a crucial step of the proof a version of the Arrow's Theorem by Mas-Colell and Sonnenschein [14] that is stated in Proposition 5 below.

In order to prove the main theorems we need to introduce some notations and definitions.

A group decision rule $F$ is a function mapping profiles of voters preferences on binary relations on $\mathcal{C}, F: \mathcal{P}^{\mathcal{V}} \longrightarrow \mathcal{B}$. A group decision rule is quasitransitive if for all $P \in \mathcal{P}^{\mathcal{V}}, F(P)$ is a quasiorder. A group decision rule is unanimous if for any $a, b \in \mathcal{C}$ for all profiles $P \in \mathcal{P}^{\mathcal{V}}$ such that $a P_{i} b$ for all $i \in \mathcal{V}, a F(P) b$ but not $b F(P) a$. A group decision rule satisfies Arrow's Independence of Irrelevant Alternatives (AIIA) if for all $P, P^{\prime} \in \mathcal{P}^{\mathcal{V}}$ such that for all $i \in \mathcal{V},\left(a P_{i} b\right.$ if and only if $\left.a P_{i}^{\prime} b\right), a F(P) b$ implies $a F\left(P^{\prime}\right) b$. Finally a group decision rule is oligarchical if there is a set of voters $S \subseteq \mathcal{V}$ such that for all $P \in \mathcal{P}^{\mathcal{V}} a F(P) b$ if and only if there is $i \in S$ with $a P_{i} b$.

Notice that the object of interest of our work are not group decision rules but voting correspondences. Nevertheless, for all $P \in \mathcal{P}^{\mathcal{V}}$, we can define the binary relation $R_{P}^{v} \in \mathcal{B}$ in the following way, for any $a, b \in C, a R_{P}^{v} b$ if and only if and only if $a \in v(\{a, b\}, P) .{ }^{22}$ Now, for any or any voting correspondence $v$, we can define an associated group decision rule $F^{v}$ in such a way that for all $P \in \mathcal{P}^{\mathcal{V}}, F^{v}(P)=R_{P}^{v}$.

Finally we include a pair of regularity conditions on voting correspondences. These conditions will become useful to establish the properties of the properties of the group decision rule

\footnotetext{
${ }^{22}$ The strict component of $R_{P}^{v}, \bar{R}_{P}^{v}$ is defined in the usual way.
} 
associated to a candidate stable voting correspondences. ${ }^{23}$

Condition 1 (Chernoff). A voting correspondence $v$ satisfies Chernoff Condition if and only if for all $P \in \mathcal{P}^{\mathcal{V}}$, for all $A, B \in 2^{\mathcal{C}} \backslash\{\varnothing\}, v(A \cup B, P) \subseteq v(A, P) \cup B$.

Condition 2 (Aizerman). A voting correspondence $v$ satisfies Aizerman Condition if and only if for all $P \in \mathcal{P}^{V} A, B \in 2^{\mathcal{C}} \backslash\{\varnothing\}, v(B, P) \subseteq A \subseteq B$ implies $v(A, P) \subseteq v(B, P)$.

Proposition 4 (Sen [21]). A voting correspondence $v$ satisfies Chernoff and Aizerman conditions if and only if $R_{P}^{v}$ is a quasiorder for all $P \in \mathcal{P}^{\mathcal{V}}$.

Proposition 5 (Mas-Colell and Sonnenschein [14]). A group decision rule F satisfies quasitransitivity, AIIA, unanimity if and only if $F$ is oligarchical.

\section{Proof of Theorem 4.}

The proof of Theorem 4 proceeds through a series of lemmata. From now on, we assume that $v$ is a strongly unanimous voting correspondence satisfying no-harm and insignificance.

We say $B$ is a restricted top set of $P$ at profile $\bar{P}$, if $B$ is a restricted top-set of $P$ and $\left.P\right|_{\mathcal{C} \backslash B}=\left.\bar{P}\right|_{\mathcal{C} \backslash B}$, we denote the set of profiles for which $B$ is a top-set at profile $\bar{P}$ as $\mathcal{P}^{\mathcal{V}}(B, \bar{P})$.

Lemma 5. Consider any arbitrary set of three candidates $\{a, b, c\} \in 2^{\mathcal{C}} \backslash\{\varnothing\}$ and an arbitrary preference profile $\bar{P} \in \mathcal{P}^{\mathcal{V}}$. Then for all $P \in \mathcal{P}^{\mathcal{V}}(\{a, b, c\}, \bar{P}) v(\mathcal{C}, P) \subseteq\{a, b, c\}$.

Proof. Pick any arbitrary $\{a, b, c\} \in 2^{\mathcal{C}} \backslash\{\varnothing\}$ and $\bar{P} \in \mathcal{P}^{\mathcal{V}}$. Consider a preference profile $P \in$ $\mathcal{P}^{\mathcal{V}}(\{a, b, c\}, \bar{P})$ such that $\{a, b\}$ is a restricted top-set. Notice that either $a \notin v(\mathcal{C}, P)$, or $b \notin v(\mathcal{C}, P)$, or $\{a, b\} \subseteq v(\mathcal{C}, P)$. Assume first, $b \notin v\left(\mathcal{C}, P^{\prime}\right)$, then by insignificance $v\left(\mathcal{C}, P^{\prime}\right)=$ $v\left(\mathcal{C} \backslash\{b\}, P^{\prime}\right)$, and by strong unanimity $v\left(\mathcal{C} \backslash\{b\}, P^{\prime}\right)=\{a\}$, and also $v\left(\mathcal{C}, P^{\prime}\right)=\{a\}$. If $\{a, b\} \subseteq$ $v(\mathcal{C}, P)$ by strong unanimity $v\left(\mathcal{C} \backslash\{a\}, P^{\prime}\right)=\{b\}$, and $v\left(\mathcal{C} \backslash\{b\}, P^{\prime}\right)=\{a\}$. By no-harm, we know $v(\mathcal{C}, P) \subseteq(v(\mathcal{C} \backslash\{a\}, P) \cup\{b\})$, and therefore $v(\mathcal{C}, P)=\{a, b\}$. As the choice of $a$ and $b$ was arbitrary, it follows that if $\{x, y\} \subset\{a, b, c\}$ is a restricted top-set of $P \in \mathcal{P}^{\mathcal{V}}(\{a, b, c\}, \bar{P})$, then $v(\mathcal{C}, P) \subseteq\{x, y\}$.

\footnotetext{
${ }^{23}$ Excellent compendia of the choice theory literature can be found in Aizeman and Aleskerov [1], Chapter 11 of Moulin [16] and Sen [21] and [22].
} 
Now, take an arbitrary $P^{\prime} \in \mathcal{P}^{\mathcal{V}}(\{a, b, c\}, \bar{P})$. Suppose there is some $x \notin\{a, b, c\} ; x \in$ $v(\mathcal{C}, P)$. Consider now $\hat{P} \in \mathcal{P}^{\mathcal{V}}\left(a, b, \bar{P}\right.$ such that $\left.P^{\prime}\right|_{\mathcal{C} \backslash\{c\}}=\left.\hat{P}\right|_{\mathcal{C} \backslash\{c\}}$. By ii) in the definition of voting correspondence, it should be the case that $v\left(\mathcal{C} \backslash\{c\}, P^{\prime}\right)=v(\mathcal{C} \backslash\{c\}, \hat{P})$. By the arguments in the previous paragraph, $v(\mathcal{C}, \hat{P}) \subseteq\{a, b\}$, and by insignificance, $v(\mathcal{C}, \hat{P})=$ $v(\mathcal{C} \backslash\{c\}, \hat{P})$. Hence, $v\left(\mathcal{C} \backslash\{c\}, P^{\prime}\right) \subseteq\{a, b\}$, and applying no-harm we obtain that, $v\left(\mathcal{C}, P^{\prime}\right) \subseteq$ $\{a, b, c\}$, contrary to our supposition.

Moreover, from similar arguments, it follows that also for any $d \in \mathcal{C}, v(\mathcal{C} \backslash\{d\}, P) \subseteq$ $\{a, b, c\} \backslash\{d\}$.

At this point, for any arbitrary triple of candidates $\{a, b, c\} \in 2^{\mathcal{C}} \backslash\{\varnothing\}$ and preference profile $\bar{P} \in \mathcal{P}^{\mathcal{V}}$, we can define an auxiliary restricted voting correspondence

$$
\hat{v}_{(\{a, b, c\}, \bar{P})}: 2^{\{a, b, c\}} \backslash\{\varnothing\} \times \mathcal{P}^{\mathcal{V}}(\{a, b, c\}, \bar{P}) \longrightarrow 2^{\{a, b, c\}} \backslash\{\varnothing\}
$$

in such a way that for all $P \in \mathcal{P}^{\mathcal{V}}(\{a, b, c\}, \bar{P})$ and for all $x \in\{a, b, c\} ; \hat{v}_{(\{a, b, c\}, \bar{P})}(\{a, b, c\}, P)=$ $v(\mathcal{C}, P)$, and $\hat{v}_{(\{a, b, c\}, \bar{P})}(\{a, b, c\} \backslash\{x\}, P)=v(\mathcal{C} \backslash\{x\}, P)$. Then, $\hat{v}_{(\{a, b, c\}, \bar{P})}$ is a well defined choice function in the restricted domain $\mathcal{P}^{\mathcal{V}}(\{a, b, c\}, \bar{P})$ and satisfies strong unanimity,no-harm and insignificance since $v$ also does. Moreover as $\hat{v}_{(\{a, b, c\}, \bar{P})}$ is always well defined, for any set of three candidates and any preference profile we can define the group decision rule $F^{\hat{v}(\{a, b, c\}, \bar{P})}$, that maps voters preference profile over the candidates $\{a, b, c\}$ to binary relations on $\{a, b, c\}$.

Lemma 6. For any $\{a, b, c\} \in 2^{\mathcal{C}} \backslash\{\varnothing\}$ and $\bar{P} \in \mathcal{P}^{\mathcal{V}}, F^{\hat{v}(\{a, b, c\}, \bar{P})}$ is quasitransitive.

Now, we are going to focus on the implications of no-harm and insignificance in an arbitrary restricted domain $\mathcal{P}^{\mathcal{V}}(\{a, b, c\}, \bar{P})$. In order to simplify the notation, from now on we refer to $\hat{v}_{(\{a, b, c\}, \bar{P})}$ simply by $\hat{v}$, and also $F^{\hat{v}(\{a, b, c\}, \bar{P})}$, by $F^{\hat{v}}$.

Proof. In order to prove this Lemma we have to check that for all $P \in \mathcal{P}^{\mathcal{V}}(\{a, b, c\}, \bar{P}), \hat{v}$ satisfies Chernoff and Aizerman conditions. Then, the result holds directly from the application of Proposition 4. Hence, consider arbitrary $P \in \mathcal{P}^{\mathcal{V}}(\{a, b, c\}, \bar{P})$ and $A, B \in 2^{\{a, b, c\}} \backslash\{\varnothing\}$ and let us check the different possibilities.

"Chernoff Condition". Notice first that Chernoff condition holds immediately from the definition of voting correspondence in the following cases: $A$ and $B$ are singletons, either $A$ 
or $B$ are $\{a, b, c\}$, and \# $(A \cup B)=2$. So suppose $(A \cup B)=\{a, b, c\}$ and either $A$ or $B$ is a singleton (say $B$ ). By no-harm, $\hat{v}(A \cup B, P) \subseteq \hat{v}(A \backslash B, P) \cup B$, and immediately we obtain $\hat{v}(A \cup B, P) \subseteq \hat{v}(A, P) \cup B$. On the other hand, from the definition of voting correspondence we also have that, $\hat{v}(A \cup B, P) \subseteq \hat{v}(B, P) \cup A$, and we have that Chernoff condition holds. Thus, the only remaining possibility is that $A$ and $B$ are both duples and $(A \cup B)=\{a, b, c\}$. In this case, there are candidates $d=(B \backslash A)$ and $e=(A \backslash B)$. Notice that no-harm and insignificance imply that $\hat{v}(A \cup B, P) \subseteq(\hat{v}(A \cup B \backslash\{d\}, P) \cup\{d\})$. Finally as $(A \cup B \backslash\{d\})=A$, and $\{d\} \subset B$, the previous expression also implies that $\hat{v}(A \cup B) \subseteq(\hat{v}(A, P) \cup B)$. Repeating the argument for the withdrawal of the candidate $e$, we have that $\hat{v}$ satisfies Chernoff condition.

"Aizerman Condition". Aizerman condition holds immediately in the cases in which the set $A$ is a singleton or the whole set of candidates $\{a, b, c\}$. Hence we only have to study the case in which $A$ is a duple. Without loss of generality, assume $A=\{a, b\}, B=\{a, b, c\}$. If $\hat{v}(\{a, b, c\}, P) \subseteq\{a, b\}$, insignificance implies that $\hat{v}(\{a, b\}, P)=\hat{v}(\{a, b, c\}, P)$. Thus we have that for all $A, B, \hat{v}(B, P) \subseteq A \subseteq B$, implies $\hat{v}(A, P) \subseteq \hat{v}(B, P)$.

Notice that as the choice of $P \in \mathcal{P}^{\mathcal{V}}(\{a, b, c\}, \bar{P})$ was arbitrary, we can apply Proposition 4 to state that for all $P \in \mathcal{P}^{\mathcal{V}}(\{a, b, c\}, \bar{P}), R_{P}^{\hat{v}}$ is a quasiorder. Then, it follows from its very definition that the group decision rule $F^{\hat{v}}$ is quasitransitive.

Lemma 7. For any $\{a, b, c\} \in 2^{\mathcal{C}} \backslash\{\varnothing\}$ and any $\bar{P} \in \mathcal{P}^{\mathcal{V}}$ the group decision rule $F^{\hat{v}(\{a, b, c\}, \bar{P})}$ is oligarchical with vetoers $S \subseteq(\mathcal{V} \backslash\{a, b, c\})$.

Proof. Consider an arbitrary profile $\bar{P} \in \mathcal{P}^{\mathcal{V}}$ and a restricted profile of the candidates $\{a, b, c\}$ such that, $P_{\{a, b, c\}}^{*} \in \mathcal{P}^{\{a, b, c\}}(\{a, b, c\}, \bar{P})$ and $a P_{a}^{*} b P_{a}^{*} c ; b P_{b}^{*} c P_{b}^{*} a$, and $c P_{c}^{*} a P_{c}^{*} b$. Now define an auxiliary group decision rule $F^{*}$ on the preferences of the voters who are not in $\{a, b, c\}$ in the following way, for all $P_{(\mathcal{V} \backslash\{a, b, c\})} \in \mathcal{P}^{\mathcal{V} \backslash\{a, b, c\}}(\{a, b, c\}, \bar{P}), F^{*}\left(P_{(\mathcal{V} \backslash\{a, b, c\})}\right)=F^{\hat{v}}\left(\left(P_{(\mathcal{V} \backslash\{a, b, c\})}, P_{\{a, b, c\}}^{*}\right)\right.$. Construct now the preference profile $P^{1} \in \mathcal{P}^{\mathcal{V}}(\{a, b, c\}, \bar{P})$ in the following way, for all $i \in$ $(\mathcal{V} \backslash\{a, b, c\}), P_{i}^{1}$ is such that $a P_{i}^{1} b P_{i}^{1} c$, while $P_{\{a, b, c\}}^{1}=P_{\{a, b, c\}}^{*}$. By strong unanimity $v\left(\mathcal{C} \backslash\{c\}, P^{1}\right)=$ $\{a\}$, and $v\left(\mathcal{C} \backslash\{a\}, P^{1}\right)=\{b\}$, and as $F^{\hat{v}}(\{a, b, c\}, \bar{P})$ is quasitransitive, it must be the case that also $v\left(\mathcal{C} \backslash\{b\}, P^{1}\right)=\{a\}$. Then, by the definition of $F^{\hat{v}}, F^{\hat{v}}\left(P^{1}\right)=P_{i}^{1}$ for any $i \in(\mathcal{V} \backslash i)$. 
Consider now, the profile $P^{2} \in \mathcal{P}^{\mathcal{V}}\left(\{a, b, c\}, \bar{P}\right.$ such that for all $i \in(\mathcal{V} \backslash\{a, b, c\}), b P_{i}^{2} c P_{i}^{2} a$, and $P_{\{a, b, c\}}^{2}=P_{\{a, b, c\}}^{*}$. Applying the same arguments that we have used for $P^{1}$ we have that $F^{\hat{v}}\left(P^{2}\right)=P_{i}^{2}$ for some $i \in(\mathcal{V} \backslash\{a, b, c\})$. Analogously, for the profile $P^{3} \in \mathcal{P}^{\mathcal{V}}(\{a, b, c\}, \bar{P}$ such that for all $i \in(\mathcal{V} \backslash\{a, b, c\}), c P_{i}^{3} a P_{i}^{3} b$, and $P_{\{a, b, c\}}^{3}=P_{\{a, b, c\}}^{*}$ we obtain $F^{\hat{v}}\left(P^{3}\right)=P_{i}^{3}$, for some $i \in(\mathcal{V} \backslash\{a, b, c\})$.

At this point, consider an arbitrary profile of preferences for the voters $\tilde{P} \in \mathcal{P}^{\mathcal{V}}(\{a, b, c\}, \bar{P})$ such that for all $i \in(\mathcal{V} \backslash\{a, b, c\}), a \tilde{P}_{i} b$, while $\tilde{P}_{\{a, b, c\}}=P_{\{a, b, c\}}^{*}$. Notice that $\left.\tilde{P}\right|_{\mathcal{C} \backslash\{c\}}=P^{1} \mid \mathcal{C} \backslash\{c\}$, then by $i i)$ in the definition of voting correspondence, $v(\mathcal{C} \backslash\{c\}, \tilde{P})=\{a\}$. By the definition of the group decision rule $F^{*}$ this implies that $a F^{*}\left(\tilde{P}_{(\mathcal{V} \backslash\{a, b, c\})}\right) b$, and not $b F^{*}\left(\tilde{P}_{(\mathcal{V} \backslash\{a, b, c\})}\right) a$. We can use the same argument for any profile in which a candidate is unanimoulsy preferred to another one by all the voters in $\left(\mathcal{V} \backslash\{a, b, c\}\right.$ to conclude that $F^{*}$ satisfies unanimity.

We have seen that $F^{*}$ satisfies unanimity on $\mathcal{P}^{\mathcal{V} \backslash\{a, b, c\}}(\{a, b, c\}, \bar{P})$, unrestricted domain, since the preferences of the relevant voters over $\{a, b, c\}$ are completely free, Arrow's Independence of Irrelevant Alternatives by ii) in the definition of voting correspondence and quasitransitivity by the quasitransitivity of $F^{\hat{v}}$. Now we are in conditions to Proposition 5 and we can state that $F^{*}$ is oligarchical. Moreover, since the preferences of $a, b$ and $c$ are fixed, the vetoers must belong to $(\mathcal{V} \backslash\{a, b, c\})$.

Now, we have to extend this partial result to all possible configuration of the preferences of candidates $a, b$ and $c$. If $a, b$ and $c$ are not voters, we are already done, since their preferences do not affect the voting correspondence, so we suppose that $\{a, b, c\}$ are also voters. Consider now $P_{\{a, b, c\}}^{* *} \in P^{\{a, b, c\}}(\{a, b, c\}, \bar{P})$ such that $a P_{a}^{* *} c P_{a}^{* *} b$, and $P_{\{b, c\}}^{* *}=P_{\{b, c\}}^{*}$. Define now the auxiliary group decision rule $F^{* *}$ in the same fashion that $F^{*}$. That is, for any $P_{(\mathcal{V} \backslash\{a, b, c\})} \in \mathcal{P}^{\mathcal{V} \backslash\{a, b, c\}}(\{a, b, c\}, \bar{P}), F^{* *}\left(P_{(\mathcal{V} \backslash\{a, b, c\})}\right)=F^{\hat{v}}\left(P_{(\mathcal{V} \backslash\{a, b, c\})}, P_{\{a, b, c\}}^{* *}\right)$. Consider now the profile $P^{3^{\prime}} \in \mathcal{P}^{\mathcal{V}}(\{a, b, c\}, \bar{P})$, such that for all $j \in \mathcal{V} \backslash a, P_{j}^{3^{\prime}}=P_{j}^{3}$ and $P_{a}^{3^{\prime}}=P_{a}^{* *}$. Notice that $\left.P^{3^{\prime}}\right|_{\mathcal{C} \backslash\{b\}}=\left.P^{3}\right|_{\mathcal{C} \backslash\{b\}}$ and $\left.P^{3^{\prime}}\right|_{\mathcal{C} \backslash\{c\}}=\left.P^{3}\right|_{\mathcal{C} \backslash\{c\}}$. By ii) of the definition of a voting correspondence, $v\left(\mathcal{C} \backslash\{b\}, P^{3^{\prime}}\right)=\{c\}, v\left(\mathcal{C} \backslash\{c\}, P^{3^{\prime}}\right)=\{a\}$ and by the quasitransitivity of $F^{\hat{v}}$, we obtain that $v\left(\mathcal{C} \backslash\{a\}, P^{3^{\prime}}\right)=\{c\}$. Then, $F^{* *}\left(P_{(\mathcal{V} \backslash\{a, b, c\})}^{3^{\prime}}\right)=P_{i}^{3}$, for some $i \in(\mathcal{V} \backslash\{a, b, c\})$. Consider finally the profile $P^{4} \in \mathcal{P}^{\mathcal{V}}(\{a, b, c\}, \bar{P})$, such that for all $i \in(\mathcal{V} \backslash\{a, b, c\}), b P_{i}^{4} a P_{i}^{4} c$, while $P_{\{a, b, c\}}^{4}=P_{\{a, b, c\}}^{* *}$. Notice that, $\left.P^{4}\right|_{\mathcal{C} \backslash\{c\}}=\left.P^{2}\right|_{\mathcal{C} \backslash\{c\}}$, and $\left.P^{4}\right|_{\mathcal{C} \backslash\{b\}}=\left.P^{1}\right|_{\mathcal{C} \backslash\{b\}}$. Following 
the previous arguments, we can see that $F^{* *}\left(P_{(\mathcal{V} \backslash\{a, b, c\})}^{4}\right)=P_{i}^{4}$ for some $i \in(\mathcal{V} \backslash\{a, b, c\})$. Repeating the same reasoning, it is easy to prove that $F^{* *}$ is unanimous. Therefore, by Proposition 5 , as $F^{* *}$ is a quasitransitive group decision rule defined in a universal domain and satisfying unanimity and AIIA, we know it is oligarchical.

Finally, we have to prove that the same voters hold veto power for $F^{*}$ and for $F^{* *}$. Assume the contrary, and without loss of generality suppose there is $i \in(\mathcal{V} \backslash\{a, b, c\})$ holding veto power over $F^{* *}$ but not over $F^{*}$. Consider the restricted profile of preferences $\hat{P}_{(\mathcal{V} \backslash\{a, b, c\})} \in$ $\mathcal{P}^{\mathcal{V} \backslash\{a, b, c\}}(\{a, b, c\}, \bar{P})$ such that for all $j \in \mathcal{V} \backslash\{a, b, c, i\}, a \hat{P}_{j} b \hat{P}_{j} c$, while $b \hat{P}_{i} a \hat{P}_{i} c$. Given that the voter $i$ is not a vetoer for $F^{*}$ but he is for $F^{* *}$, we have that:

$$
\begin{gathered}
v\left(\mathcal{C} \backslash\{c\},\left(\hat{P}_{(\mathcal{V} \backslash\{a, b, c\})}, P_{\{a, b, c\}}^{*}\right)\right)=\{a\}, \\
b \in v\left(\mathcal{C} \backslash\{c\},\left(\hat{P}_{(\mathcal{V} \backslash\{a, b, c\})}, P_{\{a, b, c\}}^{* *}\right)\right) .
\end{gathered}
$$

But this is a contradiction with ii) in the definition of the voting correspondence, since we have that $\left.\left(\hat{P}_{(\mathcal{V} \backslash\{a, b, c\})}, P_{\{a, b, c\}}^{*}\right)\right|_{\mathcal{C} \backslash\{c\}}=\left.\left(\hat{P}_{(\mathcal{V} \backslash\{a, b, c\})}, P_{\{a, b, c\}}^{* *}\right)\right|_{\mathcal{C} \backslash\{c\}}$.

We can apply the same arguments iteratively for all possible $P_{\{a, b, c\}} \in \mathcal{P}^{\{a, b, c\}}(\{a, b, c\}, \bar{P})$ to conclude that the group decision rule $F^{\hat{v}}$ is oligarchical and, of course, the vetoers $S$ belong to $(\mathcal{V} \backslash\{a, b, c\})$. Moreover, as the choice of $\{a, b, c\}$ and $P$ was arbitrary, for any triple of candidates $\{a, b, c\} \in 2^{\mathcal{C}} \backslash\{\varnothing\}$ and any preference profile $P \in \mathcal{P}^{\mathcal{V}}, F^{\hat{v}_{(\{a, b, c\}, P)}}$ is oligarchical, and the candidates $\{\mathrm{a}, \mathrm{b}, \mathrm{c}\}$ cannot be vetoers.

Consider now an arbitrary triple of candidates $\{a, b, c\} \in 2^{\mathcal{C}} \backslash\{\varnothing\}$ and a preference $\bar{P} \in \mathcal{P}^{\mathcal{V}}$. We will see now which are the consequences of the previous lemma over the original voting correspondence $v$ in the restricted domain $\mathcal{P}^{\mathcal{V}}(\{a, b, c\}, \bar{P})$. Let $S$ denote the oligarchy for $F^{\hat{v}}$, we will see they also have veto power for $v$. First we prove the necessity of item a) of Theorem 4 in this restricted domain. Notice that, item a) holds for all the agendas in which a candidate leaves the fray by the definitions of $F^{\hat{v}}$ and $S$. If there is a candidate $d \notin \operatorname{Pareto}\left(\mathcal{C}, P_{S}\right)$, there is $e \in\{a, b, c\}$, with $e P_{i} d$ for all $i \in S$. Notice now that for $f=(\{a, b, c\} \backslash\{d, e\}), v(\mathcal{C} \backslash\{f\}, P)=\{e\}$, since $S$ have veto power for $F^{\hat{v}}$, therefore by insignificance and no-harm it must be the case that $v$ $(\mathcal{C}, P) \subseteq\{e\} \cup\{f\}$, and then $d \notin v(\mathcal{C}, P)$. In order to prove item $b)$, notice that it holds from the definition of $F^{\hat{v}}$ whenever a candidate withdraws. Hence, we only have to check that a candidate 
cannot be the unique choice of the society unless she unanimously preferred by the members of $S$ to the remaining ones. Assume without loss of generality, there is a non-unanimous profile $P_{S}^{\prime} \in \mathcal{P}^{S}(\{a, b, c\}, \bar{P})$, such that $v\left(\mathcal{C}, P^{\prime}\right)=\{a\}$ but there is $i \in S$ such that $b P_{i}^{\prime} a$. As $i$ is a vetoer for $F^{\hat{v}}, b \in v\left(\mathcal{C} \backslash\{c\}, P^{\prime}\right)$. But insignificance implies that $v\left(\mathcal{C}, P^{\prime}\right)=v\left(\mathcal{C} \backslash\{c\}, P^{\prime}\right)$, which contradicts our assumption. Finally, notice that as $F^{\hat{v}}$ is oligarchical, item $c$ ) also holds trivially in the domain $\mathcal{P}^{\mathcal{V}}(\{a, b, c\}, \bar{P})$.

This concludes the proof in the case in which there are only three candidates $\{a, b, c\}$, since $\mathcal{P}^{\mathcal{V}}=\mathcal{P}^{\mathcal{V}}(\{a, b, c\}, \bar{P})$. We will prove the result for arbitrary sizes of the set of candidates using an induction argument. Before, we need to introduce a pair of lemmata.

Lemma 8. Consider any three candidates $a, b, c \in \mathcal{C}, v$ is oligarchical in $\mathcal{P}^{\mathcal{V}}(\{a, b, c\})$.

Proof. First, we extend our previous result to those profiles for which $\{a, b, c\}$ is top-set but are not uniform for the rest of alternatives. Take $\bar{P}, \tilde{P}$ such that $\{a, b, c\}$ is a top-set for both profiles, but there is $d \in \mathcal{C} \backslash\{a, b, c\}, \bar{P} \neq \tilde{P}$; but $\left.\bar{P}\right|_{\mathcal{C} \backslash\{d\}}=\left.\tilde{P}\right|_{\mathcal{C} \backslash\{d\}}$. We know, by the previous lemma, $F^{\hat{v}_{(\{a, b, c\}, \bar{P})}}$ and $F^{\hat{v}_{(\{a, b, c\}, \tilde{P})}}$ are oligarchical, and let us denote their respective oligarchies by $\bar{S}$ and $\tilde{S}$. Suppose to the contrary $\bar{S} \neq \tilde{S}$, then there is some voter $j \in \bar{S}, j \notin \tilde{S}$. Consider the profiles $P \in \mathcal{P}^{\mathcal{V}}(\{a, b, c\}, \bar{P})$ and $P^{\prime} \in \mathcal{P}^{\mathcal{V}}(\{a, b, c\}, \tilde{P})$ such that $\left.P\right|_{\mathcal{C} \backslash\{d\}}=\left.P^{\prime}\right|_{\mathcal{C} \backslash\{d\}}$ and for all $i \in \mathcal{V} \backslash j, a P_{i} b P_{i} c$, while $b P_{j} a P_{j} c$. Then, $v(\mathcal{C}, P)=\{a\}$, while $b \in v\left(\mathcal{C}, P^{\prime}\right)$. And, by no-harm and insignificance, $v(\mathcal{C} \backslash\{d\}, P)=\{a\}$, while $b \in v\left(\mathcal{C} \backslash\{d\}, P^{\prime}\right)$. However, $\left.P\right|_{\mathcal{C} \backslash\{d\}}=\left.P^{\prime}\right|_{\mathcal{C} \backslash\{d\}}$, and ii) in the definition of voting correspondence implies $v(\mathcal{C} \backslash\{d\}, P)=v\left(\mathcal{C} \backslash\{d\}, P^{\prime}\right)$, which contradicts our supposition about $\bar{S} \neq \tilde{S}$. We can repeat the argument as many times as necessary to prove the statement for any arbitrary $P \in \mathcal{P}^{\mathcal{V}}(\{a, b, c\})$, which leads to the desired result.

Lemma 9. For any $\{a, b, c\} \in 2^{\mathcal{C}} \backslash\{\varnothing\}$, $v$ is oligarchical on $\mathcal{P}^{\mathcal{V}}(\{a, b, c\})$ (with the same vetoers $S \subseteq(\mathcal{V} \backslash \mathcal{C})$ for each of these domains $).$

Proof. It is enough to consider some $\{a, b, d\}$ distinct from $\{a, b, c\}$ and show that the same voters have veto power on $\mathcal{P}^{\mathcal{V}}(\{a, b, c\})$ and $\mathcal{P}^{\mathcal{V}}(\{a, b, d\})$. By the previous lemma, we know 
there are an oligarchy $S$, which "dictates" on $\mathcal{P}^{\mathcal{V}}(\{a, b, c\})$ and an oligarchy $S^{\prime}$, which "dictates" on $\mathcal{P}^{\mathcal{V}}(\{a, b, d\})$. Suppose to the contrary, $S \neq S^{\prime}$, and $j \in\left(S^{\prime} \backslash S\right)$. Consider the profile $\hat{P} \in \mathcal{P}^{\mathcal{V}}(\{a, b, c, d\})$ such that $a=\operatorname{top}\left(\mathcal{C}, \hat{P}_{i}\right)$ for all $i \in(\mathcal{V} \backslash\{j\})$ but $b=\operatorname{top}\left(\mathcal{C}, \hat{P}_{j}\right)$, $a=\operatorname{top}\left(\mathcal{C} \backslash\{b\}, \hat{P}_{j}\right)$ and $d=$ bottom $\left(\{a, b, c, d\}, \hat{P}_{i}\right)$ for all $i \in \mathcal{V}$. Then, as $\hat{P} \in \mathcal{P}^{\mathcal{V}}(\{a, b, c\})$, $v(\mathcal{C}, \hat{P})=\{a\}$, and by insignificance we know also that $v(\mathcal{C} \backslash\{c\}, \hat{P})=\{a\}$. Now, consider the profile $P^{*}$, such that $c$ and $d$ are switched, hence $\left.\hat{P}\right|_{\mathcal{C} \backslash\{c\}}=\left.P^{*}\right|_{\mathcal{C} \backslash\{c\}}$, whereas $P^{*} \in \mathcal{P}^{\mathcal{V}}(\{a, b, d\})$. As $j \in S^{\prime} b \in v\left(\mathcal{C}, P^{*}\right)$, insignificance and no-harm imply that $b \in v\left(\mathcal{C} \backslash\{c\}, P^{*}\right)$. However, this contradicts $i i)$ in the definition of voting correspondence, since $\left.\hat{P}\right|_{\mathcal{C} \backslash\{c\}}=\left.P^{*}\right|_{\mathcal{C} \backslash\{c\}}$. We can repeat the argument for any set of three candidates to obtain the desired result.

Note that as the set of vetoers is the same for any $\{a, b, c\}$ and the candidates cannot be vetoers, this implies that if $\mathcal{V}=\mathcal{C}$ no strongly unanimous voting correspondence satisfies no-harm and insignificance.

At this point we are already at condition to extend our result to arbitrary sizes of the top-set candidates.

Induction Step There is $m \geq 3$ such that for all $B \in 2^{\mathcal{C}} \backslash\{\varnothing\}$ with $\# B \leq m$, then there is a set of voters $S \subseteq(\mathcal{V} \backslash \mathcal{C})$ such that for all $P \in \mathcal{P}^{\mathcal{V}}(B)$ and all $A \in\left\{\mathcal{C},\{\mathcal{C} \backslash\{a\}\}_{a \in \mathcal{C}}\right\}$ :

a) $v(A, P) \subseteq$ Pareto $\left(A, P_{S}\right)$.

b) Let $a \in A$. If there is $b \in A, i \in S$ with $b P_{i} a$, then $v(A, P) \neq\{a\}$.

Now we will prove that the result is also true for $\# B=m+1$.

Proof. Consider an arbitrary $B \in 2^{\mathcal{C}} \backslash\{\varnothing\}$, with $\# B=m+1$, and an arbitrary $P \in \mathcal{P}^{\mathcal{V}}(B)$.

We start proving $a$ ) for the case in which all the candidates run the election. Let $d \notin$ Pareto $\left(\mathcal{C}, P_{S}\right)$, as $\# B \geq 3$ there is $f \in B$ such that $d \notin P$ Pareto $(\mathcal{C} \backslash\{f\}, P)$. Find now, $P^{\prime} \in P(\mathcal{C} \backslash\{f\})$ with $\left.P\right|_{\mathcal{C} \backslash\{f\}}=\left.P^{\prime}\right|_{\mathcal{C} \backslash\{f\}}$. Evidently, \# $(B \backslash\{f\})=m$, then by the induction hypotheses $v\left(\mathcal{C} \backslash\{f\}, P^{\prime}\right) \subseteq$ Pareto $\left(\mathcal{C} \backslash\{f\}, P_{S}^{\prime}\right)=\operatorname{Pareto}\left(\mathcal{C} \backslash\{f\}, P_{S}\right)$. By ii) in the definition of voting correspondence $v(\mathcal{C} \backslash\{f\}, P)=v\left(\mathcal{C} \backslash\{f\}, P^{\prime}\right)$. Therefore by no-harm, we have that:

$$
v(\mathcal{C}, P) \subseteq v(\mathcal{C} \backslash\{f\}, P) \cup\{f\} \subseteq \text { Pareto }(\mathcal{C} \backslash\{f\}, P) \cup\{f\}
$$


which suffices to prove that Pareto dominated (according to the preferences of the vetoers) candidates are not elected at $P$ when all the candidates are at stake.

Now we check that the result also holds when an arbitrary candidate withdraws. Assume first $a \notin v(\mathcal{C}, P)$. Notice that if $d \notin \operatorname{Pareto}\left(\mathcal{C} \backslash\{a\}, P_{S}\right), d \notin \operatorname{Pareto}\left(\mathcal{C}, P_{S}\right)$ and by the previous paragraph, we know that $d \notin v(\mathcal{C}, P)$. But by insignificance $v(\mathcal{C}, P)=v(\mathcal{C} \backslash\{a\}, P)$, and no Pareto dominated candidate is elected. Finally, in the case in which $a \in v(\mathcal{C}, P)$, the argument applied in the previous paragraph can be replicated to prove the desired result. (Notice that if a candidate $a$ is elected, $a$ must belong to $B$ and evidently $\#(B \backslash\{a\})=m$.)

We prove $b$ ) using similar arguments. Consider the election at profile $P$ when al the candidates are at stake and assume to the contrary that there are $a, b \in \mathcal{C}$ such that $v(\mathcal{C}, P)=a$ but $b P_{j} a$ for some $j \in S$. For any $f \in(B \backslash\{a\})$, by insignificance as $f \notin v(\mathcal{C}, P) ; v(\mathcal{C} \backslash\{f\}, P)=\{a\}$. Now pick $P^{\prime \prime} \in \mathcal{P}^{\mathcal{V}}(B \backslash\{f\})$, with $\left.P\right|_{\mathcal{C} \backslash\{f\}}=\left.P^{\prime \prime}\right|_{\mathcal{C} \backslash\{f\}}$. As \# $(B \backslash\{f\})=m$, and $b P_{j}^{\prime \prime} a$, by the induction hypotheses, $v\left(\mathcal{C} \backslash\{f\}, P^{\prime \prime}\right) \neq\{a\}$, which leads to a contradiction since by ii) in the definition of voting correspondence $v(\mathcal{C} \backslash\{f\}, P)=v\left(\mathcal{C} \backslash\{f\}, P^{\prime \prime}\right)$, and then our assumption cannot be correct.

Finally, we can replicate the arguments in the proof of item $a$ ) to prove $b$ ) when a candidate withdraws. If some candidate $e \notin v(\mathcal{C}, P)$ quits, then $v(\mathcal{C}, P)=v(\mathcal{C} \backslash\{e\}, P)$. However, in the previous paragraph we have already proved that the election at $P$ cannot be a singleton if $a$ is not unanimously preferred for the vetoers. If an elected candidate leaves the ballot, the induction hypotheses applies directly to obtain the result.

In order to complete the proof of Theorem 4 we only have to show that the best candidates of the vetoers are elected when another candidate withdraws. Take $a \in \mathcal{C}$ and $P \in \mathcal{P}^{\mathcal{V}}$. Let $b=$ top $\left(\mathcal{C} \backslash\{a\}, P_{j}\right)$ for $j \in S$. Consider now $P^{*} \in \mathcal{P}^{\mathcal{V}}$, such that $\left.P\right|_{\mathcal{C} \backslash\{a\}}=\left.P^{*}\right|_{\mathcal{C} \backslash\{a\}}, a=\operatorname{top}\left(\mathcal{C}, P_{i}^{*}\right)$ for all $i \in(\mathcal{V} \backslash\{j\})$, while $a=\operatorname{top}\left(\mathcal{C} \backslash\{b\}, P^{*}\right)$. By the arguments of the induction step, we have $v\left(\mathcal{C}, P^{*}\right)=\{a, b\}$, and by no-harm $b \in v\left(\mathcal{C} \backslash\{a\}, P^{*}\right)$. Finally, by ii) in the definition of voting correspondence $v(\mathcal{C} \backslash\{a\}, P)=v\left(\mathcal{C} \backslash\{a\}, P^{*}\right)$, which yields the desired result, since the choice of $P, b$ and $j$ was arbitrary. 


\section{Proof of Theorem 1.}

Notice first that strong unanimity and unanimity are equivalent when candidates cannot vote. Moreover, by Lemma 2, we know that in this case, BDS1 candidate stability implies noharm and insignificance. Therefore, by the results in Theorem 4 , there is a set of voters, $S$, with veto power over $v$. We prove now that there can only be one such a voter, namely a dictator.

Assume to the contrary $\# S \geq 2$ and consider the preference profile $P \in \mathcal{P}^{\mathcal{V}}$ such that for some candidates $a, b, c \in \mathcal{C}, a P_{i} b P_{i} c$ for some $i \in S$, while $b P_{j} c P_{j} a$ for all $j \in(\mathcal{V} \backslash\{i\})$. By Theorem 4, we have that $v(\mathcal{C}, P)=\{a, b\}$ whereas $v(\mathcal{C} \backslash\{b\}, P)=\{a, c\}$, which is a contradiction with item i) of Lemma 2 and $v$ being BDS1 candidate stable. Hence, a unanimous BDS1 candidate stable voting correspondence only admits one vetoer, and therefore, it is dictatorial.

\section{Proof of Theorem 2.}

It is clear that dictatorial and bidictatorial voting correspondences are BDS2 candidate stable and unanimous.

Let us check sufficiency when there are only three candidates. Notice first that the voting correspondence selects just a candidate only at unanimous profiles for the vetoers. In this case, the elected candidate never improves by quitting while the non-elected ones do not affect the social choice. (The profile remains unanimous after the withdrawal of a non-elected candidate.) When $\#(v(\mathcal{C}, P))=2$, both elected candidates are not Pareto dominated, therefore if the nonelected candidate leaves, items a) and b) imply that the social choice does not change. On the other hand, if a chosen candidate withdraws, either the other selected candidate is the unique winner or the two running candidates are elected. In either case, the conditions of Lemma 3 are satisfied. Finally, if $v(\mathcal{C}, P)=\mathcal{C}$, as no candidate is Pareto dominated, for any $a \in \mathcal{C}$, $v(\mathcal{C} \backslash\{a\}, P)=(\mathcal{C} \backslash\{a\})$ and no candidate is better off after quitting.

In order to prove necessity, notice that strong unanimity and unanimity are equivalent when candidates do not vote, and BDS2 candidate stable voting correspondence satisfy no-harm and insignificance when candidates are not allowed to vote. Therefore, from Theorem 4, we know that any candidate stable under BDS2 and unanimous voting correspondence is oligarchical. We 
have just seen that the conditions of Theorem 4 are also sufficient for the case in which there are only three candidates. We will prove immediately that when there are at least four candidates, there cannot be more than two vetoers.

Assume to the contrary that a voting correspondence $v$ is BDS2 candidate stable and unanimous, but there are at least 3 voters holding veto power. Consider $\{a, b, c\} \in 2^{\mathcal{C}} \backslash\{\varnothing\}$, $P^{\prime} \in \mathcal{P}^{\mathcal{V}}(\{a, b, c\}), i, j \in S$ such that $a=\operatorname{top}\left(C, P_{i}^{\prime}\right), b=\operatorname{top}\left(\mathcal{C}, P_{j}^{\prime}\right)$ and $c=\operatorname{top}\left(\mathcal{C}, P_{k}^{\prime}\right)$ for all $k \in(\mathcal{V} \backslash\{i, j\})$. By Theorem $4, v\left(\mathcal{C}, P^{\prime}\right)=\{a, b, c\}$, and also for all $d \in(\mathcal{V} \backslash\{a, b, c\})$, $v\left(\mathcal{C} \backslash\{d\}, P^{\prime}\right)=\{a, b, c\}$. Pick now $d^{\prime} \in(\mathcal{V} \backslash\{a, b, c\}), \hat{P} \in \mathcal{P}^{\mathcal{V}}$ such that $\left.\hat{P}\right|_{\mathcal{C} \backslash\left\{d^{\prime}\right\}}=\left.P^{\prime}\right|_{\mathcal{C} \backslash\left\{d^{\prime}\right\}}$ and $d^{\prime}=\operatorname{top}\left(\mathcal{C} \backslash\{a\}, \hat{P}_{i}\right), d^{\prime}=\operatorname{top}\left(\mathcal{C}, \hat{P}_{k^{\prime}}\right)$ for all $k^{\prime} \in(\mathcal{V} \backslash\{i\})$. Then, by Theorem $4 v(\mathcal{C}, \hat{P})=$ $\{a, d\}$, while by ii) in the definition of voting correspondence $v(\mathcal{C} \backslash\{d\}, \hat{P})=\{a, b, c\}$. This contradicts item $i$ ) of Lemma 3 and it suffices to prove that $\# S \leq 2$.

Finally, we have to prove that a candidate is elected only if she is the best preferred candidate for one of the vetoers $S=\{i, j\}$. Assume to the contrary there is $P^{*} \in \mathcal{P}^{\mathcal{V}}$ such that $a=$ top $\left(\mathcal{C}, P_{i}^{*}\right)$, while $b=\operatorname{top}\left(\mathcal{C}, P_{j}^{*}\right)$ but there is $c \in(\mathcal{C} \backslash\{a, b\}), c \in v\left(\mathcal{C}, P^{*}\right)$. Then for all $d \in(\mathcal{C} \backslash\{a, b, c\}),\{a, b, c\} \subseteq v\left(\mathcal{C} \backslash\{d\}, P^{*}\right)$. Pick now $\tilde{P} \in \mathcal{P}^{\mathcal{V}},\left.\tilde{P}\right|_{\mathcal{C} \backslash\{d\}}=P^{*} \mid \mathcal{C} \backslash\{d\}$, such that $d=$ top $\left(\mathcal{C} \backslash\{a\}, \tilde{P}_{i}\right), d=\operatorname{top}\left(\mathcal{C}, \tilde{P}_{j}\right)$. By Theorem $4, v(\mathcal{C}, \tilde{P})=\{a, d\}$, while by ii) of the definition of voting correspondence, $\{a, b, c\} \subseteq v(\mathcal{C} \backslash\{d\}, \tilde{P})$, which contradicts item i) of Lemma 3 . Hence, only the best preferred candidates of the vetoers can be elected when all the candidates run the election. A similar argument applies to the agendas in which a candidate withdraws, which concludes the proof.

\section{Proof of Theorem 3.}

Again notice that strong unanimity and unanimity are equivalent when candidates cannot vote. Moreover, in Lemma 4 we have proved that leximin candidate stability is equivalent to no-harm and insignificance both together when the candidates are not voters. Then the result is a direct application of Theorem 4. 


\subsection{Appendix 2: Proof of Lemma 1}

We introduce a final piece of notation which becomes quite useful in the proof of Lemma 1. For any $a \in \mathcal{C}, P_{a} \in \mathcal{P}^{a}$ and any integer $n=1, \ldots, \# \mathcal{C}, P_{a}(n)$ refers to the candidate in the n-th position according to the preference ordering $P_{a}$. Evidently, $\operatorname{top}\left(\mathcal{C}, P_{a}\right)=P_{a}(1)$, $\operatorname{bottom}\left(\mathcal{C}, P_{a}\right)=P_{a}(\# \mathcal{C})$.

\section{Proof of Lemma 1.}

Item i) Notice that $a \notin(X \cup Y)$, then we can find $P_{a} \in \mathcal{P}^{a}$ such that for all $b \in(X \backslash Y)$, $c \in(X \cap Y)$ and $d \in(Y \backslash X), b P_{a} c P_{a} d$. Then,for an arbitrary $u_{a}$ fitting $P_{a}$, we have that

$$
\frac{1}{\# X} \sum_{x \in X} u_{a}(x)>\frac{1}{\# Y} \sum_{y \in Y} u_{a}(y)
$$

This is enough to show there is $\succsim_{a}^{2} \in \mathcal{D}_{a}^{2}$, such that $X \succ_{a}^{2} Y$.

Item ii) Assume to the contrary there is $\succsim \in \mathcal{D}_{a}^{1}$ such that $Y \succsim X$. In this case there should exist $P_{a} \in \mathcal{P}^{a}, u_{a}$ fitting $P_{a}$ and a prior probability assessment $\lambda$ such that:

$$
\sum_{y \in Y} \frac{\lambda(y) u_{a}(y)}{\left(\sum_{y^{\prime} \in Y} \lambda\left(y^{\prime}\right)\right)} \geq \frac{\lambda(a) u_{a}(a)}{\lambda(a)+\sum_{y^{\prime} \in Y} \lambda\left(y^{\prime}\right)}+\sum_{y \in Y} \frac{\lambda(y) u_{a}(y)}{\left(\lambda(a)+\sum_{y^{\prime} \in Y} \lambda\left(y^{\prime}\right)\right)}
$$

Nevertheless, (1) implies that $\sum_{y \in Y} \frac{\lambda(y) u_{a}(y)}{\left(\sum_{y^{\prime} \in Y} \lambda\left(y^{\prime}\right)\right)} \geq u_{a}(a)$ which is a contradiction with $u_{a}$ being consistent with some $P_{a}$. Therefore our assumption was false, and for all $a \in \mathcal{C}$, $X, Y \in 2^{\mathcal{C}} \backslash\{\varnothing\}$ such that $X=(Y \cup\{a\}), \succsim \in \mathcal{D}_{a}^{1}, X \succ Y$.

Item iii) Let $X, Y \in 2^{\mathcal{C}} \backslash\{\varnothing\}$, be such that $\# Y>1, a \in Y, Y \neq(X \cup\{a\})$. We can study two cases separately:

Case iii-a) There is $b \in(Y \backslash\{a\}), b \notin X$.

Case iii-b) There is some $c \in X, c \notin Y$. 
Case iii-a). Consider $b \in(Y \backslash\{a\}), b \notin X$ and find $P_{a} \in \mathcal{P}^{a}$ with $b=\operatorname{bottom}\left(\mathcal{C}, P_{a}\right)$, a prior assessment $\lambda$ such that for some $\varepsilon>0, \lambda(b)=(1-\varepsilon), \lambda(x)=\frac{\varepsilon}{\#(\mathcal{C}-1)}$ for all $x \in(\mathcal{C} \backslash\{b\})$. Construct now a utility function $u_{a}$ fitting $P_{a}$ in such a way that $u_{a}\left(P_{a}(n)\right)=1-\frac{(n-1)}{\# \mathcal{C}} \varepsilon$ for $n=1, \ldots, \# \mathcal{C}-1$, while $u_{a}\left(P_{a}(\# \mathcal{C})\right)=u_{a}(b)=0$.

Notice that, for some $\varepsilon>0$ small enough we obtain:

$$
\sum_{x \in X} \frac{\lambda(x) u_{a}(x)}{\left(\sum_{x^{\prime} \in X} \lambda\left(x^{\prime}\right)\right)}>\sum_{y \in Y} \frac{\lambda(y) u_{a}(y)}{\left(\sum_{y^{\prime} \in Y} \lambda\left(y^{\prime}\right)\right)},
$$

since the left hand side converges to 1 as $\varepsilon$ goes to 0 , while the right hand side converges to 0 as $\varepsilon$ decreases. This suffices to prove the existence of the desired ordering over sets in the case $i i i-a)$.

Case iii-b). Assume now there is $c \in X, c \notin Y$. Find $P_{a}^{\prime} \in \mathcal{P}^{a}$ such that $c=\operatorname{top}\left(\mathcal{C} \backslash\{a\}, P_{a}\right)$, prior assessment $\lambda^{\prime}$ such that for some $\varepsilon^{\prime}>0, \lambda^{\prime}(c)=\left(1-\varepsilon^{\prime}\right)$ while $\lambda^{\prime}(x)=\frac{\varepsilon^{\prime}}{\#(\mathcal{C}-1)}$ for all $x \in(\mathcal{C} \backslash\{c\})$. Finally, construct a utility function representing $P_{a}^{\prime}, u_{a}^{\prime}$ in such a way that $u_{a}^{\prime}(a)=1, u_{a}^{\prime}(c)=1-\varepsilon^{\prime}$, and $u_{a}^{\prime}\left(P_{a}^{\prime}(n)\right)=\frac{\varepsilon^{\prime}}{n}$ for all $n=3, \ldots, \# \mathcal{C}$.

For some $\varepsilon^{\prime}>0$ small enough we get again that:

$$
\sum_{x \in X} \frac{\lambda^{\prime}(x) u_{a}^{\prime}(x)}{\left(\sum_{x^{\prime} \in X} \lambda^{\prime}\left(x^{\prime}\right)\right)}>\sum_{y \in Y} \frac{\lambda^{\prime}(y) u_{a}^{\prime}(y)}{\left(\sum_{y^{\prime} \in Y} \lambda^{\prime}\left(y^{\prime}\right)\right)}
$$

since the left hand side converges to 1 , while the right hand side converges to $\frac{1}{\# Y}$, which is smaller to 1 since we assumed $\# Y>1$. Therefore, using $P_{a}^{\prime}, u_{a}^{\prime}$ and $\lambda^{\prime}$ we obtain the result for the case $i i i-b$ ), which concludes the proof of the case $i i i-b)$.

Item iv) We only have to check the case in which for some $b \in Y,(X \backslash\{a\})=(Y \backslash\{b\})$. Consider an arbitrary $P_{a} \in \mathcal{P}^{a}$. Notice that $a P_{a} b$, and moreover $\# X=\# Y$, and $(X \backslash\{a\})=$ $(Y \backslash\{b\})=(X \cap Y)$. Then for all utility function $u_{a}$ representing $P_{a}$, it holds that

$$
\frac{1}{\# X}\left(u_{a}(a)+\sum_{x \in X \cap Y} u_{a}(x)\right)>\frac{1}{\# Y}\left(u_{a}(b)+\sum_{x \in X \cap Y} u_{a}(x)\right) .
$$

As the choice of $P_{a}$ was arbitrary, this suffices to prove that for all BDS2 orderings $\succsim \in \mathcal{D}_{a}^{2}, X \succ Y$. 
Item v) Let $a \in \mathcal{C}, X, Y \in 2^{\mathcal{C}} \backslash\{\varnothing\}$ be such that $a \in Y, \# Y=1$ and for any $b \in(Y \cup\{\varnothing\})$, $(X \backslash\{a\}) \neq(Y \backslash\{b\})$. We have to study two cases:

Case v-a) There is $c \in(Y \backslash\{a\}), c \notin X$.

Case v-b) There is a set of candidates $\left\{b_{1}, \ldots, b_{k}\right\}$ such that either $X=\left(Y \cup\left\{b_{1}, \ldots, b_{k}\right\}\right)$, or $X=\left(Y \cup\left\{b_{1}, \ldots, b_{k}\right\}\right) \backslash\{a\}$ (in this case $k>1$ ).

Case v-a). Find $\hat{P}_{a} \in \mathcal{P}^{a}$ such that $c=\operatorname{bottom}\left(\mathcal{C}, \hat{P}_{a}\right)$. Construct now a utility function fitting $\hat{P}_{a}$ such that

$$
{ }_{a}(c)<\left(\frac{\# Y}{\# X} \sum_{x \in X}{ }_{a}(x)-\sum_{y \in Y}{ }_{a}(y)\right) .
$$

We can find such a ${ }_{a}$ since $c$ is the worst candidate according to the preference order $\hat{P}_{a}$. This suffices to prove the case $v-a)$.

Case v-b). Find $P_{a}^{*} \in \mathcal{P}^{a}$ such that for all $c \in \mathcal{C} \backslash\left(\left\{b_{1}, \ldots, b_{n}\right\} \cup\{a\}\right)$ and for all $l=1, \ldots, k$ $b_{l} P_{a}^{*} c$. Construct $u_{a}^{*}$ in such a way that for some $\varepsilon>0$ small enough $u_{a}^{*}\left(P_{a}^{*}(n)\right)=1-\frac{(n-1)}{\# \mathcal{C} \varepsilon}$ for all $n=1, \ldots, k+1$, while $u_{a}^{*}\left(P_{a}^{*}(n)\right)=\frac{\varepsilon}{n}$, otherwise.

If $X=\left(Y \cup\left\{b_{1}, \ldots, b_{k}\right\}\right)$, for some $\varepsilon$ sufficiently small we obtain that $\frac{1}{\# X}\left(\sum_{x \in X} u_{a}^{*}(x)\right)$ is arbitrarily close to $\frac{k+1}{\# Y+k}$, while $\frac{1}{\# Y}\left(\sum_{y \in Y} u_{a}^{*}(y)\right)$ is arbitrarily close to $\frac{1}{\# Y}$. Notice that $\frac{k+1}{\# Y+k}>\frac{1}{\# Y}$ if $\# Y>1$, and there exists $\succsim \in \mathcal{D}_{a}^{2}$, such that $X \succ Y$.

Finally if $X=\left(Y \cup\left\{b_{1}, \ldots, b_{k}\right\}\right) \backslash\{a\}$ (with $k>1$ ) we can find $\varepsilon$ small enough to get $\frac{1}{\# X}\left(\sum_{x \in X} u_{a}^{*}(x)\right)$ arbitrarily close to $\frac{k}{\# Y+k-1}$, while $\frac{1}{\# Y}\left(\sum_{y \in Y} u_{a}^{*}(y)\right)$ is again arbitrarily close to $\frac{1}{\# Y}$. In this case notice that $\frac{k}{\# Y+k-1}>\frac{1}{\# Y}$, since $\# Y$ and $k$ are both greater than 1 , and the desired conclusion holds.

\section{References}

[1] Aizerman M. and F. Aleskerov(1995), "Theory of Choice", Amsterdam, North Holland.

[2] Barberà S., W. Bossert and P. Pattanaik (2002), Ranking Sets of Objects; forthcoming in S. Barberà, P.J. Hammond and C. Seidl (eds.); Handbook of Utility Theory, Volume 2. Kluiwert Academic Publishers. 
[3] Barberà S., B. Dutta and A. Sen (2001), Strategy-Proof Social Choice Correspondences; Journal of Economic Theory 101, pp. 374-394.

[4] Berga D., G. Bergantiños, J. Massó and A. Neme (2002), Voting by Committees with Exit; mimeo Universitat Autònoma de Barcelona.

[5] Besley T. and S. Coate (1997), An Economic Model of Representative Democracy; Quarterly Journal of Economics 112, pp. 85-114.

[6] Campbell D. and J.S. Kelly (2000), A Trade-Off Result for Preference Revelation; Journal of Mathematical Economics 34, pp. 129-141.

[7] Ching S., and L. Zhou (2002), Non-Manipulable Multi-Valued Social Choice Rules; Social Choice and Welfare 19, pp. 569-580.

[8] Demange G. (1987), Non-manipulable Cores; Econometrica 55, pp. 1057-1074.

[9] Denicolò V. (1987), Some Further Results on Non-binary Social Choice; Social Choice and Welfare 4, pp. 277-285.

[10] Dutta B., M.O. Jackson and M. Le Breton (2001), Strategic Candidacy and Voting Procedures; Econometrica 69, pp 1013-1038.

[11] Ehlers L. and J. Weymark (2003), Candidate Stabilility and Non-Binary Choice, Economic Theory 22, pp. 233-243.

[12] Eraslan H., A. McLennan, Strategic Candidacy and Multivalued Voting Procedures; mimeo, University of Pennsylvania and University of Minnesota (2002).

[13] Gärdenfors P. (1976), Manipulation of Social Choice Functions; Journal of Economic Theory 13, pp. 217-228.

[14] Mas-Colell A. and H. Sonnenschein (1972), General Possibility Theorems for Groups Decision; Review of Economic Studies 39, pp. 185-192. 
[15] Miller N. (1980), A New Solution Set for Tournaments and Majority Voting: Further Graphical Theoretical Approaches to the Theory of Voting; Americal Journal of Political Science 24, pp. 68-69.

[16] Moulin H. 1988), "Axioms in Collective Decision Making"; Cambridge, Cambridge University Press.

[17] Osborne M.J. and A. Slivinski (1996), A Model of Political Competition with Citizen Candidates; Quarterly Journal of Economics 111, pp. 65-96.

[18] Pattanaik P.K. (1973), On the Stability of Sincere Voting Situations; Journal of Economic Theory 6, pp. 558-574.

[19] Pattanaik P.K. (1978), "Strategy and Group Choice"; Amsterdam, New Holland.

[20] Rodríguez-Álvarez C. (2002), Candidate Stability and Probabilistic Voting Procedures; mimeo, Universitat Autònoma de Barcelona.

[21] Sen A.K. (1971), Choice Functions and Revealed Preference; Review of Economic Studies 38, pp. 307-317.

[22] Sen A.K. (1977), Social Choice: A Reexamination;Econometrica 45, pp. 53-90.

[23] Sönmez T. (1997), Manipulation Via Capacities in Two-Sided Matching Models; Journal of Economic Theory 77, pp. 197-204. 